\title{
Admissibility and Refutation Some Characterisations of Intermediate Logics
}

\author{
Jeroen P. Goudsmit
}

November 25th 2013, revised March 27th, July 4th and July 28th 2014

\begin{abstract}
Refutation systems are formal systems for inferring the falsity of formulae. These systems can, in particular, be used to syntactically characterise logics. In this paper, we explore the close connection between refutation systems and admissible rules.

We develop technical machinery to construct refutation systems, employing techniques from the study of admissible rules. Concretely, we provide a refutation system for the intermediate logics of bounded branching, known as the Gabbay-de Jongh logics. We show that this gives a characterisation of these logics in terms of their admissible rules. To illustrate the technique, we also provide a refutation system for Medvedev's logic.

\section{Keywords}

Intermediate Logic, Admissible Rules, Refutation, Gabbay-de Jongh Logic, Medvedev’s Logic
\end{abstract}

According to Łukasiewicz (1951), we assert true propositions, and reject false ones. He remarked that rejection had been neglected in the study of formal logic, and introduced a formal system to inductively derive rejections of false propositions. We call such systems refutation systems, following Scott (1957) and Skura (1990). The general theory of such systems has been studied extensively by Słupecki, Bryll, and Wybraniec-Skardowska $(1971,1972)$. In this paper, we focus on refutation systems for intermediate logics.

A refutation system can be thought of as a proof system for rejection. Instead of deriving that one can correctly assert a statement through a series of truth-preserving inferences from given axioms, as one does in a proof system of assertion, one derives the refutability of a propositional statement through a series of non-truth preserving inferences from given anti-axioms. Proofs in a refutation system will be called refutations, and a formula will be called refutable whenever a refutation exists ending in this formula.

Let us, by way of example, present a reformulation of the original refutation system for the classical propositional calculus (CPC) as given by Łukasiewicz (1951). This particular presentation, and all the following, will be in the style of Skura (1992), which goes back to Scott (1957). ${ }^{1}$ Here $x$

"This paper appeared in the Archive for Mathematical Logic. The final publication is available at Springer via http: //dx.doi.org/10.1007/s00153-014-0388-5.

${ }^{1}$ See Słupecki and Bryll (1973) for a similar presentation and further pointers to the literature. 
denotes a propositional variable, $\phi$ and $\psi$ both denote propositional formulae, and $\sigma$ denotes a substitution.

$$
\frac{\dashv}{\dashv x} \mathrm{Ax} \quad \frac{\dashv \sigma(\phi)}{\dashv \phi} \text { Subs } \frac{\dashv \psi \quad \phi \vdash_{\mathrm{CPC}} \psi}{\dashv \phi} \mathrm{MT}
$$

This refutation system is both sound (all refutable formulae are not derivable in CPC) and complete (all formulae that are not derivable in CPC are refutable). Skura (1999, Section 1.2) gives a thorough proof of completeness, let us simply remark that to each classically non-derivable formula there is a substitution under which it is equivalent to falsity, whence completeness is clear. $^{2}$

Gödel (1932) showed that the intuitionistic propositional calculus (IPC) enjoys the disjunction property. With this observation in hand, Eukasiewicz (1952) proposed the following refutation system for IPC, which he conjectured to be complete.

$$
\frac{\dashv x}{\dashv x} \quad \frac{\dashv \sigma(\phi)}{\dashv \phi} \text { Subs } \quad \frac{\dashv \psi \quad \phi \vdash_{\mathrm{IPC}} \psi}{\dashv \phi} \mathrm{MT} \quad \frac{\dashv \phi \quad \dashv \psi}{\dashv \phi \vee \psi} \mathrm{DP}
$$

Kreisel and Putnam (1957) proved that this system is not complete by constructing an intermediate logic, a consistent axiomatic extension of IPC, now known as KP. This logic KP has the disjunction property, and it is unequal to IPC, falsifying the conjecture. There exist, in fact, uncountably many intermediate logics with the disjunction property, see Chagrov and Zakharyaschev (1991) for a survey.

Observe that the rules DP and MT are structural, in the sense that every substitution instance of an instance of this rule is again an instance of this rule. This does not hold for Ax and Subs, and necessarily so, as we will argue in Section 1.

Scott (1957) gave a refutation system which is both sound and complete for IPC by replacing DP with RScott, as given in Fig. 1. The rule RScott, however, has several side-conditions which makes it inherently non-structural, in that it has instances with invalid substitution instances. The Kleene (1962)-Kleene (1962) slash suggests that the refutation system obtained by replacing DP with RKleene might be complete. This is indeed the case, as follows from de Jongh (1968, Chapter IV). ${ }^{3}$ Another refutation system was given by Dutkiewicz (1989) based on the semantic tableaux of E. W. Beth. Neither of these rules are structural.

Another approach was taken by Skura (1989), who introduced the rules RSkura of Fig. $1{ }^{4}$ The rule scheme he proposed was structural, and bears great resemblance to the so-called Visser rules, a rule scheme central to the study of admissibility in intermediate logics.

\footnotetext{
${ }^{2}$ See also Lemma 13 for more details.

${ }^{3}$ We refer to Bezhanishvili (2004) for a proof using the universal model, and to Iemhoff (2001a, Proposition 5.1) for a proof using admissible rules. These authors do not propose such a refutation system, the rule given here is taken from Skura (1999, Section 5.4).

${ }^{4}$ Traces of this rule can be seen in Wroński (1973, Theorem 4).
} 


$$
\begin{gathered}
\frac{\dashv\left(\bigwedge_{i=1}^{n} \chi_{i} \rightarrow \phi_{i}\right) \rightarrow \chi_{i} \text { per } i \text { such that } \phi_{i} \text { is no variable }}{\dashv\left(\bigwedge_{i=1}^{n} \chi_{i} \rightarrow \phi_{i}\right) \rightarrow x} \text { RScott with } \phi_{1}, \ldots, \phi_{n} \neq \top \\
\frac{\dashv \psi \rightarrow \chi \text { per } \chi \in \Delta}{\dashv \psi \rightarrow \bigvee \Delta} \text { RKleene with } \psi \mid \psi \\
\frac{\dashv\left(\bigwedge_{i=1}^{n} \chi_{i} \rightarrow \phi_{i}\right) \rightarrow \chi_{j} \text { per } j=1, \ldots, n}{\dashv\left(\bigwedge_{i=1}^{n} \chi_{i} \rightarrow \phi_{i}\right) \rightarrow \bigvee_{j=1}^{n} \chi_{j}} \text { RSkura } \\
\frac{\dashv(\bigvee \Delta \rightarrow \phi) \rightarrow \chi \operatorname{per} \chi \in \Delta}{\dashv(\bigvee \Delta \rightarrow \phi) \rightarrow \bigvee \Delta} \mathrm{RD}
\end{gathered}
$$

Figure 1: Refutation rules for IPC

The admissible rules of a logic can be thought of as those rules under which the set of its theorems is closed. Such rules were covered explicitly by Lorenzen (1955), although they appeared earlier, for instance in the work of Johansson (1937). In CPC, all admissible rules are derivable, but this is not the case for IPC. An early example of a non-derivable but admissible rule of IPC is the following rule due to Harrop (1960), known as independence of premise.

$$
\neg \chi \rightarrow \phi \vee \psi /(\neg \chi \rightarrow \phi) \vee(\neg \chi \rightarrow \psi)
$$

Prucnal (1979) proved that this rule is admissible for all intermediate logics. ${ }^{5}$ Later, Minari and Wroński (1988) proved that, in the above, $\neg \chi$ can be replaced by an arbitrary Harrop formula, all the while maintaining admissibility. Problem 40 of Friedman (1975) states that the set of admissible rules of IPC is decidable, which has been proven by Rybakov (1984). Iemhoff (2001b) and Rozière (1992) independently proved that the Visser rules are sufficient to derive all single-conclusion admissible rules of IPC. This result was later generalised to the the logics of bounded branching by Goudsmit and Iemhoff (2014).

In this paper, we show that the study of admissible rules is closely related to the study of refutation systems. In particular, we provide a refutation system for the logics of bounded branching $\mathrm{T}_{n}$, also known as the Gabbay-de Jongh logics, making use of admissible rules similar to those given by Goudsmit and Iemhoff (2014). The method naturally extends to cover IPC, providing an intimate connection between the characterisation of IPC in terms of its multi-conclusion admissible rules, as given by Iemhoff (2001a), and the structural refutation system of Skura (1989). Moreover, the same machinery applies to Medvedev's Logic, re-proving a result by Skura (1992).

\footnotetext{
${ }^{5}$ In this paper, Prucnal also solved problem 41 of Friedman (1975). This problem, rephrased in modern language, conjectured that there exists an intermediate logic with the disjunction property for which all admissible rules are derivable. The logic Prucnal used was Medvedev's logic. See Wojtylak (2004) for an exposition of this result, and see Grigolia (1995) for an algebraic proof. We revisit Medvedev's logic in Section 3.
} 


\section{Preliminaries}

We work over the full propositional language on a set of variables $X$ defined by the following Backus-Nauer form:

$$
\mathcal{L}(X)::=\top|\perp| X|\mathcal{L}(X) \wedge \mathcal{L}(X)| \mathcal{L}(X) \vee \mathcal{L}(X) \mid \mathcal{L}(X) \rightarrow \mathcal{L}(X)
$$

Sets of variables will be denoted by $X, Y$ and variables by their lower-case variants. Formulae will be denoted $\phi, \psi, \chi, \theta$ and sets of formulae by $\Gamma, \Pi, \Delta, \Theta$. Given a finite set of formulae $\Delta$ we write $\Lambda \Delta$ and $\bigvee \Delta$ for the iterated conjunction and disjunction of $\Delta$, in the understanding that $\Lambda \emptyset=\top$ and $\bigvee \emptyset=\perp$. We define a rule to be a pair of finite sets of formulae, written $\Gamma / \Delta$.

We wish not to be concerned with the intricacies of different axiomatisations of logics. In order to avoid this, we make use of multi-conclusion consequence relations. We refer to Wójcicki (1988) for background on consequence relations, and to Iemhoff (2013) for an in-depth description of the use of consequence relations surrounding admissible rules. The multi-conclusion variant we use here is taken from Scott $(1971,1974)$. The main difference with the definition in Cintula and Metcalfe (2010) is that we do not assume structurality in a consequence relation.

1 Definition (Multi-conclusion Consequence Relation)

A set of rules $\vdash$ is called a multi-conclusion consequence relation whenever it is closed under the following for all finite sets of formulae $\Gamma, \Pi, \Delta, \Theta$ and formulae $\psi$. Here we write $\Gamma \vdash \Delta$ to mean $(\Gamma / \Delta) \in \vdash$.

$$
\begin{aligned}
& 1 \text { If } \Gamma \cap \Delta \neq \emptyset \text { then } \Gamma \vdash \Delta \text {; } \\
& 2 \text { If } \Pi \subseteq \Gamma, \Theta \subseteq \Delta \text { and } \Pi \vdash \Theta \text { then } \Gamma \vdash \Delta \text {; } \\
& 3 \text { If } \Gamma \vdash \psi, \Delta \text { and } \Gamma, \psi \vdash \Delta \text { then } \Gamma \vdash \Delta .
\end{aligned}
$$

The above three conditions, and the corresponding conditions in Definition 3, are respectively called reflexivity, weakening and transitivity. Whenever $\Gamma \vdash \Delta$ entails $\sigma(\Gamma) \vdash \sigma(\Delta)$ for all substitutions $\sigma$, we say that the consequence relation $\vdash$ is structural. We think of consequence relations as describing derivability, so if $\Gamma \vdash \Delta$ we say that $\Delta$ is derivable from $\Gamma$. When we say that a formula $\phi$ is derivable, we mean that $\{\phi\}$ is derivable from $\emptyset$. Such formulae we also call theorems.

Given any set of rules $\mathcal{R}$, one can consider the least consequence relation containing these rules, which we will denote by $\vdash_{\mathcal{R}}$. When $\mathcal{R}$ is structural, in the sense that it is closed under substitutions, it is clear that $\vdash_{\mathcal{R}}$ is structural as well. This is easy to see when we think of $\vdash_{\mathcal{R}}$ as being inductively defined, with the base-case explicating that $\vdash_{\mathcal{R}}$ is an extension of $\mathcal{R}$, and the inductive cases ensuring closure under the three conditions of Definition 1.

By IPC we mean the set of theorems of intuitionistic propositional logic, see for instance Troelstra and Dalen (1988). An intermediate logic $\mathrm{L}$ is a proper subset of the set of all formulae, that contains IPC, and that satisfies:

1 If $\phi \in \mathrm{L}$ and $\sigma$ is a substitution then $\sigma(\phi) \in \mathrm{L}$; 
2 If $\phi \in \mathrm{L}$ and $\phi \rightarrow \psi \in \mathrm{L}$ then $\psi \in \mathrm{L}$.

In particular, IPC is an intermediate logic. We refer to the first rule as being closed under substitutions, and the latter as being closed under modus ponens.

To each intermediate logic $L$ we associate a multi-conclusion consequence relation $\vdash_{\mathrm{L}}$, as below in (1). Note that this consequence relation is structural. We can see this as a special case of the relation $\vdash_{\mathcal{R}}$ described above, when we let $\mathcal{R}$ be the set of all rules $\Gamma / \Delta$ satisfying the right-hand condition in (1) below.

$$
\Gamma \vdash_{\mathrm{L}} \Delta \text { if and only if } \bigwedge \Gamma \rightarrow \bigvee \Delta \in \mathrm{L}
$$

There can be many consequence relations with the same set of theorems. We, for instance, could also consider the consequence relation defined by the following, and the set of theorems would be exactly the same as that of $\vdash_{\mathrm{L}}$.

$$
\Gamma \vdash \Delta \text { if and only if } \bigwedge \Gamma \rightarrow \chi \in L \text { for some } \chi \in \Delta .
$$

In this paper we focus on multi-conclusion admissible rules, as first suggested by Kracht (1999), and used in the context of modal logic by Jeřábek (2005) and intermediate logics by Goudsmit (2013a). Admissible rules, as defined by the aforementioned authors, are inherently structural. We first define refutation rules much like Skura (2009), ${ }^{6}$ and using this definition we define admissible rules. Note that our notion of a refutation rule has both multiple conclusions and multiple assumptions. For our purposes we quickly will forget the multiple assumptions, as for intermediate logics this difference is immaterial.

2 Definition (Refutation Rules \& Admissible Rules)

Let $\vdash$ be a consequence relation. We say that $\Gamma / \Delta$ is a refutation rule with respect to $\vdash$ if $\vdash \chi$ holds for some $\chi \in \Delta$ when $\vdash \phi$ holds for all $\phi \in \Gamma$. A rule is said to be an admissible rule, denoted $\Gamma \vdash \Delta$, when $\sigma(\Gamma) / \sigma(\Delta)$ is a refutation rule for all substitutions $\sigma$.

Naturally, $r$ itself is again a multi-conclusion consequence relation. Given any set of refutation rules $\mathcal{R}$ one can consider $\vdash_{\mathcal{R}}$, and it can be readily proven that all rules in $\vdash_{\mathcal{R}}$ are refutation rules with respect to $\vdash$. As remarked above, when $\mathcal{R}$ is structural then $\vdash_{\mathcal{R}}$ is structural as well. These observations combine to show that $\sim$ is structural. Also note that if $\vdash$ is structural, then $r$ is an extension of $\vdash$.

In the following few paragraphs, we will show how the system of refutation rules for CPC, as given in the introduction, can be seen as a kind of consequence relation. Let us first define $\mathcal{R}$ as

$$
\Delta \mathcal{R} \phi \text { iff if } \dashv \chi \text { for all } \chi \in \Delta \text { then } \dashv \phi .
$$

Consider MT, which states that $\dashv \psi$ and $\phi \vdash_{\mathrm{CPC}} \psi$ entail $\dashv \phi$. From this observation, it naturally follows that if $\phi \vdash_{\mathrm{CPC}} \psi$ then $\psi / \phi$ is contained in $\mathcal{R}$. It is also easy to see that the reversal of this rule, $\phi / \psi$, is a refutation rule with respect to $\vdash_{\mathrm{CPC}}$. In a similar manner Subs corresponds to the rule $\sigma(\phi) / \phi$, which is an element of $\mathcal{R}$, and its reversal is a refutation rule. One can also see that

\footnotetext{
${ }^{6}$ A similar definition appeared earlier in Skura (2005, Section 3), where it was called a generalised Socratic rule.
} 
$x / \emptyset$ is a valid refutation rule by virtue of $x$, or any variable for that matter, being non-derivable in

$\vdash_{\mathrm{CPC}}$. This rule, of course, corresponds to Ax.

From the above, we can conclude that the rules of the refutation system of CPC correspond to refutation rules in the formal sense of Definition 2. Moreover, see that $R$ satisfies the singleconclusion variants of Definition 1, as given in Definition 3.

3 Definition (Single-conclusion Consequence Relation)

A relation $\vdash$ between finite sets of formulae and formulae is called a consequence relation whenever the following hold for all finite sets of formulae $\Gamma, \Pi, \Delta$ and formulae $\chi$ and $\theta$.

$$
\begin{aligned}
& 1 \chi \vdash \chi ; \\
& 2 \text { If } \Gamma \subseteq \Pi \text { and } \Pi \vdash \chi \text { then } \Gamma \vdash \chi \text {; } \\
& 3 \text { If } \Gamma \vdash \chi \text { and } \Delta, \chi \vdash \theta \text { then } \Gamma, \Delta \vdash \theta .
\end{aligned}
$$

As the above argument suggests, it makes good sense to define a refutation system with respect to $\vdash$ as being a single-conclusion consequence relation $R$ such that:

$$
\text { if } \Delta R \phi \text { then } \phi / \Delta \text { is a refutation rule with respect to } \vdash \text {. }
$$

Analogously to the situation with multiple-conclusion consequence relations, one can define a least single-conclusion consequence extending a given set of rules $\mathcal{R}$. When we take $\mathcal{R}$ to be the set of all rules $\Delta / \phi$ satisfying (3) then Lemma 1 below shows that $\mathcal{R}$ itself is a single-conclusion consequence relation.

\section{Lemma}

Let $\vdash$ be a (multi-conclusion) consequence relation, let $\mathcal{R}$ be a set of rules each satisfying (3) and let $\vdash_{\mathcal{R}}$ be the least single-conclusion consequence relation extending $\mathcal{R}$. All rules in $\vdash_{\mathcal{R}}$ satisfy (3). In particular, the reverse of the totality of all refutation rules is a consequence relation.

Proof By induction along the inference of $\Delta R \phi$, we prove that $\phi / \Delta$ is a refutation rule. The base case follows by assumption. We only treat the case of transitivity. Suppose $\Delta_{1}, \theta R \phi$ and $\Delta_{2} R \theta$ with $\Delta=\Delta_{1} \cup \Delta_{2}$. By induction, we know both $\phi /\left(\Delta_{1} \cup\{\theta\}\right)$ and $\theta / \Delta_{2}$ to be refutation rules. Assume that $\vdash \phi$ holds. This ensures us that either $\vdash \theta$ or $\vdash \chi$ for some $\chi \in \Delta_{1}$. In the former case, it follows that $\vdash \chi$ for some $\chi \in \Delta_{2}$. Hence $\vdash \chi$ for some $\chi \in \Delta$ holds in both cases, finishing the argument.

We call a refutation system complete whenever $\forall \phi$ is equivalent to $\dashv \phi$, for all formulae $\phi$. In symbols, $\dashv=\not$. Following Słupecki, Bryll, and Wybraniec-Skardowska (1971), we say that a logic is $\mathrm{E}$-decidable when it has a complete refutation system.

In Definition 4 below, we construct refutation systems out of a basic set of "falsehoods" (or antiaxioms) $\Theta$ and a set of rules $\mathcal{R}$. These are the types of structural systems we wish to consider. Do note that the reverse of $\vdash_{\mathrm{Rf}}$ is not structural in the sense of consequence relations, and indeed, for our purposes, never could be. For whenever $\vdash$ is a consequence relation corresponding to an intermediate logic, it is clear that variables must be refutable, yet anything is a substitution instance of a variable. 
As remarked above Lemma 1, the reversal of the rules in a refutation system ought to be refutation rules. It is clear that the conditions (1)-(3) all satisfy this, hence Lemma 1 guarantees that $\vdash_{\mathrm{Rf}}$ indeed meets this requirement.

\section{Definition}

Let $\vdash$ be a (multi-conclusion) consequence relation, let $\Theta$ be a set of non-derivable formulae and let $\mathcal{R}$ be a set of admissible rules. The refutation system determined by $\Theta$ and $\mathcal{R}$ is the least singleconclusion consequence relation $\vdash_{\mathrm{Rf}}$ such that the following hold.

$1 \emptyset \vdash_{\mathrm{Rf}} \theta$ for all $\theta \in \Theta$;

$2 \sigma(\phi) \vdash_{\mathrm{Rf}} \phi$ for all formulae $\phi$ and all substitutions $\sigma$;

$3 \Delta \vdash_{\mathrm{Rf}} \phi$ for all $\phi \mathcal{R} \Delta$.

The above definition can be reformulated in the same form as the refutation system given in the introduction, as we state in Lemma 2 below. We will use this form throughout the following, silently sidestepping the single-conclusion consequence relation formulation of Definition 4. One can think of the relation $\vdash_{\mathrm{Rf}-1}$, defined within the proof below, as a formalised version of the relation $\dashv$ of Lemma 1. Intuitively speaking, whereas in Lemma 1 we consider all refutation rules, in Lemma 2 we restrict ourselves to those rules where the refutation can be "witnessed formally" by the predicate $\dashv(-)$.

\section{Lemma}

Let the situation be as in Definition 4 and inductively define the predicate $\dashv(-)$ on formulae as below. We now have $\emptyset \vdash_{\text {Rf }} \phi$ precisely if $\dashv \phi$ for any formula $\phi$.

$$
\frac{\theta \in \Theta}{\dashv \theta} \text { Ax } \quad \frac{\dashv \sigma(\phi)}{\dashv \phi} \text { Subs } \quad \frac{\dashv \chi \operatorname{per} \chi \in \Delta \quad \phi / \Delta \in \mathcal{R}}{\dashv \phi} \operatorname{Inv} \phi / \Delta
$$

Proof By structural induction along the definition of $\dashv(-)$, it immediately follows that if $\dashv \phi$ then $\emptyset \vdash_{\mathrm{Rf}} \phi$. Indeed, in the base case we have $\dashv \phi$ because $\phi \in \Theta$, so $\emptyset \vdash_{\mathrm{Rf}} \phi$ holds by definition. Now suppose that $\dashv \phi$ holds because of Subs. Induction ensures us that $\emptyset \vdash_{\mathrm{Rf}} \sigma(\phi)$, and we know that $\sigma(\phi) \vdash_{\mathrm{Rf}} \phi$ holds as well. Through transitivity, as provided by Definition 3.(3), it is immediate that $\emptyset \vdash_{\mathrm{Rf}} \phi$.

To prove the other direction, we first define an auxiliary relation $\vdash_{\mathrm{Rf} \dashv}$ as follows:

$$
\Delta \vdash_{\mathrm{Rf} \dashv} \phi \text { iff if } \dashv \chi \text { for all } \chi \in \Delta \text { then } \dashv \phi .
$$

The desired will be obtained through the following two claims. Indeed, assuming both claims it is clear that if $\emptyset \vdash_{\mathrm{Rf}} \phi$ then $\emptyset \vdash_{\mathrm{Rf} \dashv} \phi$ by (1), and so $\dashv$ by (2). It is clear that (2) holds, and the proof of (1) is similar to a contrapositive formulation of Lemma 1.

$1 \vdash_{\mathrm{Rf}}$ is a subset of $\vdash_{\mathrm{Rf} \dashv}$;

$2 \emptyset \vdash_{\mathrm{Rf} \dashv} \phi$ holds precisely if $\dashv \phi$ for any formula $\phi$. 
To prove results on admissibility, it is often convenient to reason semantically. For this purpose we use Kripke models, see e.g. Troelstra and Dalen (1988) or Chagrov and Zakharyaschev (1997). A Kripke model on a set of variables $X$ is a partial order $K$ together with a monotone map $v$ : $K \rightarrow \mathbf{P} X$, called a valuation, where $\mathbf{P} X$ denotes the set of subsets of $X$ ordered by inclusion. We define $v, k \Vdash \phi$ for formulae $\phi \in \mathcal{L}(X)$ and nodes $k \in K$ as usual. Given a node $k \in K$ we write $\operatorname{Th}(v, k)$ for the theory of this node, that is, the set of $\phi \in \mathcal{L}(X)$ such that $v, k \Vdash \phi$. We abbreviate $\bigcap_{k \in K} \operatorname{Th}(v, k)$ as $\operatorname{Th}(v, K)$. We will also write $\operatorname{Th}(K)$ to mean the intersection of all $\operatorname{Th}(v, K)$ for sets $X$ and monotone maps $v: K \rightarrow \mathbf{P} X$.

When there is little chance of confusion, we simply say that $K$ is a model, and omit reference to both $X$ and $v$. Maps between models on $X$ are, as usual, bounded morphisms or p-morphisms. For partial orders $P$ we write $W \uparrow$ for $W \subseteq P$ to mean the upset generated by $W$, that is to say, the set $\{p \in P \mid w \leq p$ for some $w \in W\}$. Similarly we write $W \Uparrow$ to mean the strict upset generated by $W$, defined as $W \uparrow-W$. A subset $W \subseteq P$ is said to be an anti-chain when all elements are pairwise incomparable. We write $\max W$ for the set of maximal elements in $W$, where we consider an element $k$ to be maximal if $k \uparrow=\{k\}$.

We will make heavy use of the machinery described by Goudsmit (2013b). Much of this is folklore, for more background and references to the literature we refer to the aforementioned. The main definitions and results we depend upon are given below, with sparse motivation.

5 Definition (Cover)

Let $K$ be a model, $W \subseteq K$ and $k \in K$. We say that $W$ covers $k$, written $W \kappa k$, when $k \uparrow=$ $W \uparrow \cup\{k\}$.

\section{Definition}

Let $v: K \rightarrow \mathbf{P} X$ be a model. We say that $K$ is concrete when for every pair $a, b \in K$ we have that $a=b$ whenever both $v(a)=v(b)$ and for all $k \in K-\{a, b\}$ one has $a \leq k$ if and only if $b \leq k$. The model $K$ is image finite when for all $k \in K$ the set $k \uparrow$ is finite. Finally, we call $K$ refined when for all $a, b \in K$ we have a $\phi \in \mathcal{L}(X)$ such that $a \Vdash \phi$ and $b \| \phi$ whenever $a \not \leq b$.

Let us give some pointers to the use of these definitions in the context of this paper. It is easy to verify that every refined model is concrete, the converse holds for image-finite models by Theorem 2 below. Indeed, if $a, b \in K$ are such that $v(a)=v(b)$, and $a \leq k$ holds precisely if $b \leq k$ for all $k \in K-\{a, b\}$, then one can easily show that $\operatorname{Th}(a)=\operatorname{Th}(b)$. This immediately proves $a=b$ whenever $K$ is refined. Also note that every map whose domain is a refined model has to be injective. As a consequence, concrete image-finite models (over a set of variables) are isomorphic to rooted subsets of the universal model of IPC (over that same set of variables) as defined in Theorem 1. From this observation, Lemma 5 is quite easy to obtain. For a proof we refer to Goudsmit (2013b, Corollary 4).

\section{Theorem}

Let $X$ be a finite set of variables. There is a model $\mathrm{U}_{\mathrm{L}}(X)$ of $\mathrm{L}$, called the universal model of $\mathrm{L}$ over $X$, which is such that to any rooted finite model $K$ of $\mathrm{L}$ there is a unique map $f: K \rightarrow \mathrm{U}_{\mathrm{L}}(X)$. This model $\mathrm{U}_{\mathrm{L}}(X)$ is concrete, and its theory consists of the $\mathrm{L}$-theorems in $\mathcal{L}(X)$.

Below we introduce the Jankov (1963)-de Jongh (1968). We refer to up $k$ as the "upset formula" and nd $k$ as the "not-downset formula" of $k$. When $K$ is a rooted model, we sometimes write up $K$ 
and nd $K$ to refer to the upset- and not-downset formula of the root of $K$. A proof of Theorem 2, in the notation employed here, can be found in Goudsmit (2013b, Theorem 2), cf. Bezhanishvili (2006, Section 3.3).

\section{Definition (Characteristic Formulae)}

Let $v: K \rightarrow \mathbf{P} X$ be an image-finite model. Per node $k \in K$ we define:

$$
\begin{aligned}
\operatorname{props} k & :=\{p \in X \mid k \Vdash p\}, \\
\text { news } k & :=\{p \in X \mid k \Uparrow \Vdash p \text { and } k \Downarrow p\} .
\end{aligned}
$$

Now define maps up (-), nd (-) $: K \rightarrow \mathcal{L}(X)$ by well-founded recursion as follows, where $W$ denotes the set of immediate successors of $k$.

$$
\begin{aligned}
& \operatorname{up} k:=\bigwedge \operatorname{props} k \wedge\left(\left(\bigvee \text { news } k \vee \bigvee_{w \in W} \text { nd } w\right) \rightarrow \bigvee_{w \in W} \text { up } w\right), \\
& \text { nd } k:=\operatorname{up} k \rightarrow \bigvee_{w \in W} \text { up } w .
\end{aligned}
$$

We will write up $U$ to mean $\bigvee_{k \in U}$ up $k$.

\section{Theorem}

For any concrete image finite model $K$ and all $a, b \in K$ we have $b \Vdash$ up $a$ iff $a \leq b$ and $b \Downarrow$ nd $a$ iff $b \leq a$.

\section{Corollary}

Let $k$ be a node in $\mathrm{U}_{\mathrm{IPC}}(X)$. We have $k \| \phi$ precisely if $\phi \vdash_{\mathrm{IPC}}$ nd $k$. Moreover, $\nvdash \mathrm{CPC}$ nd $k$ if and only if $k$ is maximal.

Proof Note that, for each $\phi \in \mathcal{L}(X)$, we have $\vdash_{\mathrm{CPC}} \phi$ precisely if $m \Vdash \phi$, for all maximal $m \in$ $\mathrm{U}_{\mathrm{IPC}}(X)$. From here, the desired is immediate.

\section{Lemma}

Let $v: K \rightarrow \mathbf{P} X$ be a finite concrete model. To every model $w: K \rightarrow \mathbf{P} Y$ there is a substitution $\sigma: \mathcal{L}(Y) \rightarrow \mathcal{L}(X)$ such that $v, k \Vdash \sigma(\phi)$ iff $w, k \Vdash \phi$ for all $k \in K$.

Proof For convenience, we write $\operatorname{coz}_{u}(\phi):=\{k \in K \mid u, k \Vdash \phi\}$ for $u=v, w$. This is the set of elements of $K$ which, under the valuation $u$, make the formula $\phi$ true. We define $\sigma$ on variables as below. This definition is well-defined due to the finiteness of $K$, which ensures that $\operatorname{coz}_{w}(\phi)$ is finite and hence definable by means of a Jankov-de Jongh formula.

$$
\sigma(x):=\operatorname{upcoz}_{w}(\phi)
$$

Let us now prove, by structural induction along $\phi$, that

$$
\operatorname{coz}_{v}(\sigma(\phi))=\operatorname{coz}_{w}(\phi)
$$

For propositional variables, this much is clear. Indeed, $k \in \operatorname{coz}_{v}(\sigma(\phi))$ holds precisely if $k \in$ $\operatorname{coz}_{w}(\phi)$. Let us treat the implicative case where $\phi=\psi \rightarrow \chi$, all other cases can be proven 
similarly. Consider the following chain of equivalences, where the second equivalence is through Theorem 2, the fourth through induction and the final one again through Theorem 2.

$$
\begin{aligned}
k \in \operatorname{coz}_{v}\left(\operatorname{upcoz}_{w}(\psi \rightarrow \chi)\right) & \text { iff } v, k \Vdash \operatorname{up} \operatorname{coz}_{w}(\psi \rightarrow \chi) \\
& \text { iff } w, k \Vdash \psi \rightarrow \chi \\
& \text { iff } w, l \Vdash \psi \text { implies } w, l \Vdash \chi \text { for all } l \geq k \\
& \text { iff } v, l \Vdash \sigma(\psi) \text { implies } w, k \Vdash \sigma(\chi) \text { for all } l \geq k \\
& \text { iff } k \in \operatorname{coz}_{v}\left(\operatorname{up} \operatorname{coz}_{w}(\psi) \rightarrow \operatorname{up} \operatorname{coz}_{w}(\chi)\right)
\end{aligned}
$$

The following lemma illustrates that one can endow any partial order with a concrete model. By using the elements of the order as variables, all elements can easily be distinguished, making the proof of concreteness straightforward.

\section{Lemma}

Let $K$ be a partial order. Define the valuation $v_{K}$ as below. The model $v_{K}: K \rightarrow \mathbf{P} K$ is concrete.

$$
v_{K}: K \rightarrow \mathbf{P} K, \quad k \mapsto k \downarrow:=\{l \in K \mid l \leq k\} .
$$

The following lemma occurs in several places surrounding refutability, let us mention but a few. For intermediate logics, see Skura (1989, Lemma 1), Skura (1990), Skura (1992, Theorem 2.2) and Skura (1999, Section 4.2), a similar lemma for modal logics is covered in Skura (1994, Lemma 3.1) and Goranko (1994, Lemma 1.3.3).

\section{Lemma}

Let $K$ be a finite rooted partial order $K$. For every formula $\phi \in \mathcal{L}(X)$, we have $K \forall \phi$ precisely if there is a substitution $\sigma: \mathcal{L}(X) \rightarrow \mathcal{L}(K)$ such that $\sigma(\phi) \vdash_{\text {IPC }}$ nd $v_{K}$.

Proof Let $K$ be a partial order and consider the concrete model $v_{K}: K \rightarrow \mathbf{P} K$ of Lemma 4 . As a consequence $K$ is isomorphic to $k \uparrow \subseteq \mathrm{U}_{\operatorname{IPC}}(K)$ for some $k \in \mathrm{U}_{\mathrm{IPC}}(K)$, as indicated above. Suppose that $v: K \rightarrow \mathbf{P} X$ is such that $v \forall \forall$. By Lemma 3 we know of a substitution $\sigma: \mathcal{L}(X) \rightarrow$ $\mathcal{L}(K)$ that $v_{K} \forall \sigma(\phi)$. This entails $k \forall \sigma(\phi)$, and so by Corollary 1 we obtain $\sigma(\phi) \vdash_{\mathrm{IPC}}$ nd $k$. As nd $v_{K}=$ nd $k$, we have thus proven the desired.

The following lemma will be used to prove Lemma 11, a crucial step towards Theorem 6, in which we present a refutation system for Medvedev's logic. We include the proof because the implication from (1) to (2) is a good illustration of the use of Jankov-de Jongh formulae, and it provides some of the flavour of the arguments to come in Section 2. From this lemma, the characterisation as given by Maksimova (1986, Lemma 7) naturally follows through Lemma 4.

\section{Lemma}

Let $v: K \rightarrow \mathbf{P} X$ be an image-finite model and let $n \in \mathbb{N}$ be a natural number. Now (2) implies (1), and the converse holds whenever $v$ is concrete.

1 For all $\phi \in \mathcal{L}(X)$ and $\Delta \subseteq \mathcal{L}(X)$ with $|\Delta| \leq n$, we have

$$
K \Vdash\left(\neg \phi \rightarrow \bigvee_{\chi \in \Delta} \neg \chi\right) \rightarrow \bigvee_{\chi \in \Delta} \neg \phi \rightarrow \neg \chi
$$


2 For all $W \subseteq \max K$ with $|W| \leq n$ and all $a \in K$ such that $W \subseteq \max a \uparrow$, there exits a $b \geq a$ such that $W=\max b \uparrow$.

Proof Suppose that (2) does not hold, and assume that (1) does hold. This yields some $W \subseteq \max K$ with $|W| \leq n$ and an $a \in K$ with $W \subseteq \max a \uparrow$ such that, for all $b \geq a$, we have $\max b \uparrow \neq W$. As a consequence, we know that for all $b \geq a$ that whenever $b \not \leq c$ for all $c \in \max K-W$ then $W \nsubseteq \max b \uparrow$. Define $\phi:=$ up $(\max K-W)$ and $\Delta:=\{$ up $w \mid w \in W\}$. Observe that, for any $k \in K$, we have the following, as follows directly through Theorem 2 .

$$
\begin{aligned}
& k \Vdash \neg \phi \text { if and only if } k \not \leq c \text { for all } c \in \max K-W \\
& k \Vdash \bigvee_{\chi \in \Delta} \neg \chi \text { if and only if } W \not \leq \max k \uparrow
\end{aligned}
$$

From here, (4) and (5) yield some $w \in W$ such that $a \Vdash \neg \phi \rightarrow \neg$ up $w$. Because $a \leq w$ and $w \Downarrow \phi$ and $w \Vdash$ up $w$, we reached a contradiction, proving (1) to not hold, as desired.

Now assume that $v$ is concrete and that (2) holds. Let $\phi \in \mathcal{L}(X)$ and $\Delta \subseteq \mathcal{L}(X)$ with $|\Delta| \leq n$ be given. We proceed by contradiction, so we assume that there is an $a \in K$ with

$$
a \Vdash \neg \phi \rightarrow \bigvee_{\chi \in \Delta} \neg \chi \text { yet } a \Vdash \bigvee_{\chi \in \Delta} \neg \phi \rightarrow \neg \chi
$$

The latter ensures the existence of $b_{\chi} \geq a$ such that $b_{\chi} \Vdash \neg \phi$ and $b_{\chi} \forall \neg \chi$ per $\chi \in \Delta$. Given $\chi \in \Delta$, we see that there must be a $w_{\chi} \in \max b_{\chi} \uparrow$ with $w_{\chi} \Vdash \chi$. Define $W:=\left\{w_{\chi} \mid \chi \in \Delta\right\}$ and note that $W \subseteq \max K$ and $|W| \leq n$.

It is clear that $W \subseteq \max a \uparrow$, and so, by assumption, we know of a $b \geq a$ such that $W=\max b \uparrow$. From here it is clear that $b \Vdash \neg \phi$, so from (6) we know $b \Vdash \bigvee_{\chi \in \Delta} \neg \chi$. This gives us some $\chi \in \Delta$ such that $b \Vdash \neg \chi$. As a consequence $w_{\chi} \Vdash \neg \chi$, which is blatantly fase, proving (1).

\section{Admissible Rules}

The consequence relation $\vdash_{\text {IPC }}$ has an associated relation of admissibility $r$. It is easy to see that $\perp \vdash \emptyset$. Indeed, $\vdash_{\mathrm{IPC}} \sigma(\perp)$ simply does not hold for any $\sigma$, so this rule is vacuously admissible. This is is an example of what Rybakov, Terziler, and Gencer (1999) call a passive rule. Note that this rule is, in our definition of $\vdash_{\text {IPC }}$, both derivable and admissible. Another example of a rule is $x \vee y /\{x, y\}$. This rule is admissible for $\vdash_{\mathrm{IPC}}$, and the argument to show this uses the well-known fact that IPC has the disjunction property.

Consider again the independence of premise rule IPR, as covered in the introduction, listed in Fig. 2. A straightforward argument can show its non-derivability, a Kripke model with but three nodes suffices. Intuitively, this rule allows for the distribution of an implication from a negative formula over a disjunction. IPR was generalised by Harrop (1960, Theorem 3.1) to allow for a wider class of formulae on the left-hand side. Both of these results have been generalised from IPC to arbitrary intermediate logics, the former by Prucnal (1979) and the latter by Minari and Wroński (1988). 
The following rule was shown to be both admissible and non-derivable for IPC by Mints (1976).

$$
(x \rightarrow z) \rightarrow x \vee y /((x \rightarrow z) \rightarrow x) \vee((x \rightarrow z) \rightarrow y)
$$

Citkin (1977) generalised it by taking a disjunction with a fixed variable in both the assumption and conclusion. This amounts to what is now known as the first Visser rule, see Citkin (2012) for more details. Moreover, Citkin (1979a,b) presented these now so-called Visser rules, and conjectured that all admissible rules of IPC follow from them. ${ }^{7}$ D. H. J. de Jongh and A. Visser formulated the Visser rules in the eighties, but they did not publish their formulation until much later. ${ }^{8}$ Rozière (1992) and Iemhoff (2001a) independently proved that these rules suffice to derive all admissible rules of IPC. In this section, we discuss variants of the Visser rules as given by Iemhoff (2005, Section 4).

A list of the variants of the Visser rules we wish to consider is given in Fig. 2. The scheme $\mathrm{V}_{n}^{-}$gives what are known as the restricted Visser rules (cf. Iemhoff (2005)). From Iemhoff (2005, Theorem 4.7) and Theorem 3 below it follows that all of these rules are admissible precisely if $\mathrm{D}_{n}^{-}$are admissible for all $n \in \mathbb{N}$. We consider the variants $\mathrm{D}_{n}^{-}$, $\mathrm{D}_{n}$, and $\overline{\mathrm{D}}_{n}$, because these correspond to the semantic properties introduced by Iemhoff (2005). Moreover, they stratify smoothly along the intermediate logics of bounded branching. Also note that the admissibility of $\overline{\mathrm{D}}_{n}$ is equivalent to the property $P_{n}$ of Iemhoff (2001a, Definition 2).

$$
\begin{aligned}
& \left(\bigvee_{i=1}^{n} z_{i} \rightarrow x\right) \rightarrow \bigvee_{j=1}^{n} z_{j} / \bigvee\left\{\left(\bigvee_{i=1}^{n} z_{i} \rightarrow x\right) \rightarrow z_{j} \mid j=1, \ldots, n\right\} \quad\left(\mathrm{D}_{n}^{-}\right) \\
& y \vee\left(\bigvee_{i=1}^{n} z_{i} \rightarrow x\right) \rightarrow \bigvee_{j=1}^{n} z_{j} / y \vee \bigvee\left\{\left(\bigvee_{i=1}^{n} z_{i} \rightarrow x\right) \rightarrow z_{j} \mid j=1, \ldots, n\right\} \quad\left(\mathrm{D}_{n}\right) \\
& \left(\bigvee_{i=1}^{n} z_{i} \rightarrow x\right) \rightarrow \bigvee_{j=1}^{n} z_{j} /\left\{\left(\bigvee_{i=1}^{n} z_{i} \rightarrow x\right) \rightarrow z_{j} \mid j=1, \ldots, n\right\} \quad\left(\overline{\mathrm{D}}_{n}\right) \\
& \left(\bigwedge_{i=1}^{n}\left(z_{i} \rightarrow x_{i}\right)\right) \rightarrow z_{n+1} \vee z_{n+2} / \bigvee\left\{\left(\bigwedge_{i=1}^{n}\left(z_{i} \rightarrow x_{i}\right)\right) \rightarrow z_{j} \mid j=1, \ldots, n\right\} \quad\left(\bigvee_{n}^{-}\right) \\
& \neg z \rightarrow(x \vee y) / \bigvee\{\neg z \rightarrow x, \neg z \rightarrow y\}
\end{aligned}
$$

Figure 2: Several schemes of rules, all admissible for IPC.

In many situations, admissibility of some of the above rules corresponds quite nicely to certain semantic properties. Below we give these semantic properties. Note that the latter two relate intimately to the weak extension property and the offspring property of Iemhoff (2005, Section 2.5) respectively, as can be seen through an argument similar to that in Corollary 3. A deep discussion of this is beyond the scope of this paper, but sufficed to say that through Goudsmit (2013b, Corollary 6) it is clear that the class of models of an intermediate logic $L$ has the weak extension property precisely if its canonical model is weakly $n$-ary covered for all $n \in \mathbb{N}$.

One can read Lemma 7 and Theorem 3 with $K$ instantiated as $\mathrm{U}_{\mathrm{L}}(X)$. Given that $\mathrm{L}$ has the finite model property, it is easy to see that the implications of Lemma 7 then neatly correspond to the

\footnotetext{
${ }^{7}$ For a historical note on these developments we refer to Citkin (2008).

${ }^{8}$ Traces of the Visser rules can be seen in Visser (1984, Theorem 1.1.11). We refer to Visser (2002) for the Visser rules in the context of Heyting Arithmetic (his Theorem 9.1) and more historical information (his Section 1.4).
} 
previously discussed rule schemes, as spelled out in Corollary 2. In this lies the appeal of the properties of Definition 8; their validity in the universal model is exactly reflected by the admissibility of a scheme of admissible rules.

8 Definition (Model Properties)

We say that a model is:

1 downwards directed when each finite set of elements has a lower bound;

2 weakly $n$-ary covered when each set of size at most $n$ with a lower bound is a cover of some node;

3 strongly $n$-ary covered when each set of size at most $n$ with a lower bound, say $k$, is a cover of some node, say $l$, and there is a lower bound of $l$ and $k$.

\section{Lemma}

Let $K$ be a model. The following hold.

1 If $K$ is downwards directed then $K \Vdash \bigvee \Delta$ implies $K \Vdash \chi$ for some $\chi \in \Delta$;

2 If $K$ is weakly $n$-ary covered then for all $\Delta$ and $\phi$ with $|\Delta| \leq n$ we have that $K \Vdash$ $(\bigvee \Delta \rightarrow \phi) \rightarrow \bigvee \Delta$ implies $K \Vdash \bigvee_{\chi \in \Delta}(\bigvee \Delta \rightarrow \phi) \rightarrow \chi$;

3 If $K$ is strongly $n$-ary covered then for all $\Delta$ and $\phi$ with $|\Delta| \leq n$ we have that $K \Vdash \psi \vee$ $(\bigvee \Delta \rightarrow \phi) \rightarrow \bigvee \Delta$ implies $K \Vdash \psi \vee \bigvee_{\chi \in \Delta}(\bigvee \Delta \rightarrow \phi) \rightarrow \chi$

Proof Assume that $K$ is downwards directed, and suppose $\Delta$ is a set of formulae such that $K \forall \chi$ for all $\chi \in \Delta$. Per $\chi \in \Delta$, this yields a $w_{\chi} \in K$ such that $w_{\chi} \forall \chi$. By downwards directness, we know there to be a $k \in K$ such that $k \leq w_{\chi}$, for all $\chi \in \Delta$. If $k \Vdash \bigvee \Delta$ then $k \Vdash \chi$ for some $\chi \in \Delta$, and as such, $w_{\chi} \Vdash \chi$ by construction. Via this contradiction, we arrive at (1).

Now suppose that $K$ is strongly covered, and take $\Delta$ and $\phi, \psi$ to be such that $K \forall \psi \vee \bigvee_{\chi \in \Delta}(\bigvee \Delta \rightarrow \phi) \rightarrow$ $\chi$ and $|\Delta| \leq n$. This gives us some $k \in K$ with $k \forall \psi$, and $k \forall \bigvee_{\chi \in \Delta}(\bigvee \Delta \rightarrow \phi) \rightarrow \chi$. The latter yields $w_{\chi} \geq k$ such that $w_{\chi} \Vdash \bigvee \Delta \rightarrow \phi$, yet $w_{\chi} \Vdash \forall \chi$. See that $W:=\left\{w_{\chi} \mid \chi \in \Delta\right\}$ has a lower bound, namely $k$. It is also quite clear that $|W| \leq n$. As $K$ is strongly $n$-ary covered this yields $l, l^{\prime} \in K$ with $W \kappa l$, and $l^{\prime} \leq l, k$. Observe that $l \not \forall(\bigvee \Delta \rightarrow \phi) \rightarrow \bigvee \Delta$, whence (3) is clear. We omit a proof of (2), the reader will be able to reconstruct a proof of this statement from the above argument.

\section{Theorem}

Let $K$ be an image finite and concrete model. All implications of Lemma 7 are equivalences.

Proof For (1), let $W \subseteq K$ be of size at most $n$. Observe that $w \forall$ nd $w$, and as such, $K \forall \forall$ up $w$ for all $w \in W$. By assumption, it follows that $K \forall \bigvee_{w \in W}$ nd $w$. This yields a $k \in K$ such that $k \Downarrow$ nd $w$, which in turn precisely proves that $k$ is a lower bound for $W$.

We now treat (3), see Goudsmit (2013b, Theorem 3) for a proof of (2). Suppose that $W \subseteq K$ is finite and has a lower bound, say $k$. For convenience define

$$
\Delta:=\{\text { nd } w \mid w \in W\}, \phi:=\bigvee_{w \in W} \text { up } w, \psi:=\operatorname{nd} k
$$


We can see that $k \forall \psi \vee \bigvee_{\chi \in \Delta}(\bigvee \Delta \rightarrow \phi) \rightarrow \chi$. Indeed, both disjuncts are clearly falsified at $k$. Remark that $|\Delta| \leq n$. By assumption, this ensures the existence of some $l^{\prime} \in K$ such that

$$
l^{\prime} \forall \psi \vee(\bigvee \Delta \rightarrow \phi) \rightarrow \bigvee \Delta .
$$

Because $l^{\prime}$ falsifies the left-hand disjunct we know $l \leq k$. Similarly, because $l^{\prime}$ falsifies the righthand disjunct, we obtain a $l \geq l^{\prime}$ such that $W \kappa l$ by Goudsmit (2013b, Corollary 7), where $K$ is to be read as $l^{\prime} \uparrow$. This proves the desired.

Corollary 2 below follows immediately from the above Theorem 3 and the completeness of universal models, as stated in Theorem 1. See Corollary 3 as an illustration of how the "internal" relation of being downwards directed relates to a well-known property of the models of a logic, cf. Maksimova (1986, Theorem 1) and Gabbay and de Jongh (1974, Lemma 14).

2 Corollary

Let $L$ be an intermediate logic with the finite model property. Now $L$ has the disjunction property precisely if its universal models are downwards directed. Moreover, $L$ admits $D_{n}^{-}$(or $D_{n}$ ) precisely if its universal models are weakly (or strongly) $n$-ary covered.

3 Corollary

Any intermediate logic $\mathrm{L}$ with the finite model property has the disjunction property precisely if to every pair of finite rooted models $K_{1}$ and $K_{2}$ there is a rooted model $K$ such that there are nodes $k_{1}, k_{2} \in K$ and morphisms $f_{i}: K_{i} \rightarrow k_{i} \uparrow$ per $i=1,2$.

Proof We show only the implication from left to right, the other direction is of similar difficulty. Suppose that $\mathrm{L}$ has the disjunction property and let $K_{1}$ and $K_{2}$ be arbitrary models. Without loss of generality, we may assume that $K_{1}$ and $K_{2}$ are models on the same set of variables $X$. By Theorem 1 we know of $k_{1}, k_{2} \in \mathrm{U}_{\mathrm{L}}(X)$ such that $f_{i}: K_{1} \rightarrow k_{i} \uparrow$ exist per $i=1,2$. We know $\mathrm{L}$ to have the disjunction property, so Corollary 2 ensures us that $\mathrm{U}_{\mathrm{L}}(X)$ is downwards directed. This yields a $k \in \mathrm{U}_{\mathrm{L}}(X)$ such that $k_{1} \uparrow, k_{2} \uparrow \subseteq k \uparrow$. We take $K:=k \uparrow$, and this completes the argument.

A basis of admissibility is, intuitively, a set of admissible rules from which all others follow. We define this notion formally below.

9 Definition (Basis of Admissible Rules)

A set of admissible rules $\mathcal{R} \subseteq \vdash$ is a basis of admissibility $\vdash_{\mathcal{R}}$ equals $\vdash$.

\section{Remark}

When $\mathcal{R}_{1}$ and $\mathcal{R}_{2}$ are sets of admissible rules such that $\vdash_{\mathcal{R}_{1}}=\vdash_{\mathcal{R}_{2}}$ it is clear that the structural refutation system determined by $\Theta$ and $\mathcal{R}_{1}$ equals that determined by $\Theta$ and $\mathcal{R}_{2}$ for any set of formulae $\Theta$. In particular, if $\mathcal{R}$ is a basis of admissible rules then the structural refutation system determined by $\Theta$ and $\mathcal{R}$ equals that determined by $\Theta$ and $r$. As such, basis of admissibility are of interest in constructing succinct structural refutation systems. 


\section{Admissible Approximations}

Consider an arbitrary intermediate logic $L$ and its consequence relation $\vdash_{L}$ as a preorder on the set of propositional formulae on a fixed, countably infinite, set of variables. When considering $r_{L}$ as a preorder in a similar manner, it becomes apparent that the former is a sub-preorder of the latter. Consequently, one can wonder whether this inclusion functor has a left adjoint. Ghilardi (1999, p. 874) proved that such a left adjoint exists in the case where L is IPC. When this left-adjoint exists we call the image of a formula $\phi$ under it the admissible approximation of $\phi{ }^{9}$

Admissible approximations are useful in proving a certain set of rules to be a basis of admissibility. For examples of the use of this concept see Iemhoff (2001b, Theorem 3.13), Jeřábek (2005, Theorem 4.5), Jeřábek (2010, Theorem 3.8), Goudsmit and Iemhoff (2014, Theorem 3), and Goudsmit (2013b, Lemma 20). In this section, we illustrate how admissible approximations come into play when constructing refutation systems. We introduce a very particular type of model, the admissible approximation of the not-downset formulae of which will be a disjunction of classically non-derivable formulae.

It should be noted that the results we obtain about admissible approximations are not necessary, strictly speaking, to obtain our end-results on refutability. We do include them here to drive home the idea that bases of admissibility and refutation systems can be quite closely related.

Recall that to each partial order one can assign a valuation such that the resulting model is concrete, as shown in Lemma 4. The valuation there is such that all elements of the underlying order are assigned distinct variables. In Definition 10, we specify a kind of partial order for which we can construct a more "economic" valuation, taking values only in the maximal nodes of $K$. This valuation will assign to an element the set of maximal elements above it. There are situations in which this will suffice to distinguish between all elements, for instance when the underlying partial order is a proper tree, as shown in Lemma 8.

The advantage of such a valuation will become clear by Lemma 14, very roughly speaking, the formulae up $k$ and nd $k$ will be of a form amenable to manipulation by the earlier described admissible rules. See Fig. 3 for examples and non-examples of the notions introduced in the following definition. The partial order $K_{1}$ is maximally separable and $K_{1}, K_{2}$ and $K_{3}$ are maximally distinguishable. Note that $K_{2}, K_{3}$ and $K_{4}$ are most certainly not maximally separable, and the latter is not maximally distinguishable.

10 Definition (Maximally Separable and Distinguishable)

Let $K$ be a partial order. We say that $K$ is maximally separable when the following equivalence holds for all $a, b \in K$

$$
a \leq b \text { iff } \max b \uparrow \subseteq \max a \uparrow .
$$

\footnotetext{
${ }^{9}$ We deviate a little from the currently prevalent use of the term "admissible approximation" in doing this. More commonly, as for instance in Jeřábek (2010, Definition 3.6) and Goudsmit and Iemhoff (2014, Definition 19), a close connection to "projective approximations" as introduced by Ghilardi (1999) is maintained. An admissible approximation of $\phi$ thus commonly is defined to be a set of formulae $\Delta$ satisfying certain properties such that $\bigvee \Delta$ is an admissible approximation in the above sense. We do not need these additional properties in the following exposition, hence our sloppiness in employing this term.
} 

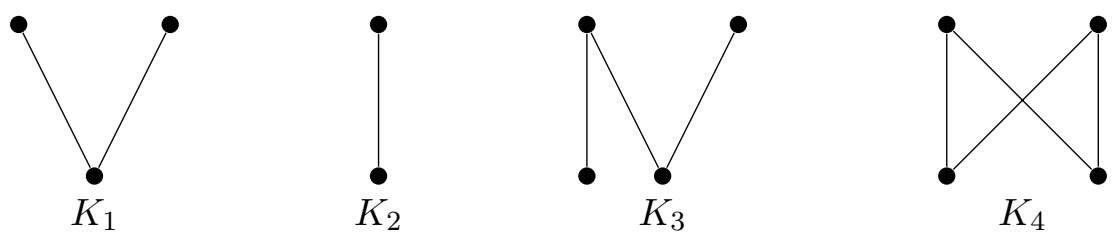

Figure 3: Examples of maximal separability and distinguishability.

We call $K$ maximally distinguishable when the model $\mathrm{m}_{K}$ defined below is concrete.

$$
\mathrm{m}_{K}: K \rightarrow \mathbf{P} \max K, \quad k \mapsto\{k\} \cap \max K
$$

Recall that IPC is complete with respect to the Jaśkowski sequence, a particular sequence of proper trees, see Smoryński (1973) and Surma, Wroński, and Zachorowski (1975) for more details. We point out that proper finite trees are maximally separable. ${ }^{10}$ To be completely precise, a tree is a rooted partial order $T$ such that for all $a, b, c \in T$ with $a, b \leq c$ we have $a \leq b$ or $b \leq a$. A tree $T$ is said to be proper when $a \kappa b$ if and only if $a=b$ for all $a, b \in T$.

\section{Lemma}

Every finite tree is maximally separable if and only if it is proper.

Proof Let $T$ be a finite tree, and suppose that $T$ is proper. Note that if $a, b \in T$ are such that $b<a$ then there is a $m \in \max T$ such that $b \leq m$ yet $a \not \leq m$. Indeed, suppose that $b<a$, and let $W$ be the set of immediate successors of $b$. Because of properness we know there to be an $a^{\prime} \in W$ with $a^{\prime} \neq a$. Choose a $m \in \max T$ such that $a^{\prime} \leq m$. If $a \leq m$ then $a^{\prime}$ and $a$ are comparable because $T$ is a tree, a contradiction.

Now suppose that $a, b \in T$ are such $\max b \uparrow \subseteq \max a \uparrow$ and assume that $a \not \subset b$. There must be be a maximal $m \in \max T$ with $b \leq m$, and so $a, b \leq m$ holds by assumption. Because $T$ is a tree, it follows that $a \leq b$ or $b \leq a$, which yields $b<a$. By the above paragraph, there is a $m^{\prime} \in \max T$ with $b \leq m^{\prime}$ and $a \not \leq m^{\prime}$, a contradiction. This proves that $T$ is maximally separable.

Now assume that $T$ is maximally separable and that $T$ is not proper. This yields $a, b \in T$ with $a \kappa b$ and $a \neq b$. Consider any $m \in \max T$ and assume $b \leq m$. Because $a \kappa b$, we know that either $a \leq m$ or $b=m$. In the latter case, we obtain a contradiction because $m<a$, and $m$ is maximal. By maximal separability, we now know that $a \leq b$, yet $b \leq a$ was known. Consequently $a=b$ follows, a contradiction, proving the desired.

\section{Lemma}

Let $K$ be a maximally separable partial order, and endow it with the valuation $\mathrm{m}_{K}$. For each $a, b \in K$ we have

$$
a \leq b \text { iff for all } m \in \max K-a \uparrow \text { it holds that } b \Vdash \neg m \text {. }
$$

In particular $K$ is maximally distinguishable.

\footnotetext{
${ }^{10}$ More generally speaking, any meet-semilattice where each element can be expressed as a meet of maximal elements is maximally separable.
} 
Proof The implication from left to right is clear. Indeed, suppose $a \leq b$ and take some $k \geq b$ such that $k \Vdash m$ with $m \in \max K-a \uparrow$. It follows that $k=m$, and so $b \leq m$. By maximal separability, this yields $a \leq m$, a contradiction.

In order to prove the other direction, we assume the right-hand side and take $m \in \max K$ to be such that $b \leq m$. If $a \not \leq m$ then $b \Vdash \neg m$ and so $b \Vdash \perp$, a contradiction. This proves $a \leq m$ as desired, so maximal separability proves $a \leq b$.

For the final statement, assume $a, b \in K$ are such that $\mathrm{m}_{K}(a)=\mathrm{m}_{K}(b)$ and for all $k \in K-\{a, b\}$ we have $a \leq k$ if and only if $b \leq k$. It is easy to verify that for each formula $\phi$ we have $a \Vdash \phi$ precisely if $b \Vdash \phi$. The desired is now immediate by the equivalence proven above.

10 Lemma

Let $K$ be a concrete and image-finite model on $X$, let $k \in K$ be given, and let $W \subseteq K$ be an anti-chain such that $W \kappa k$ and $|W| \neq 1$. We now know

$$
\vdash_{\mathrm{IPC}}\left(\operatorname{up} k \rightarrow \bigvee_{w \in W} \mathrm{nd} w\right) \equiv \operatorname{nd} k \text {. }
$$

Proof By Theorem 1, we know $K$ to correspond to a node in $\mathrm{U}_{\operatorname{IPC}}(X)$, for convenience's sake we use the same name for the node in $K$ and its corresponding node in $\mathrm{U}_{\mathrm{IPC}}(X)$. Through Theorem 2, we need to prove that the following are equivalent for $a \in \mathrm{U}_{\mathrm{IPC}}(X)$.

1 For all $b \geq a$ we have that if $k \leq b$ then $b \not \leq w$ for some $w \in W$.

$2 a \not \leq k$.

Suppose (1) holds and $a \leq k$. Filling in $b:=k$ in (1) yields $k \not \leq w$ for some $w \in W$, this contradiction proves (2) to hold.

Conversely, assume that (2) holds, and suppose there is a $b \geq a$ with $k \leq b$ and $b \leq w$ for all $w \in W$. Because $k \leq b$ and $W \kappa k$, we know $k=b$ or $b \in W \uparrow$. In the former case, we arrive at $k \geq a$, contradicting $a \not \leq k$. The latter case, there is a $w_{+} \in W$ such that $w_{+} \leq b$. We have $b \leq w_{+}$by assumption, and so $b \in W$ follows.

There must be a node $w_{-} \in W$ with $w_{-} \neq b$. Yet we know $b \leq w_{-}$, violating the assumption that $W$ is an anti-chain. All cases reach a contradiction, so (1) follows.

Before we continue along our main line of reasoning, let us first spend a few words on Medvedev's logic. The purpose of this intermezzo is to motivate Definition 12. In Theorem 5 we show that this definition can be used to define complete refutation systems, which we use to obtain our end-result, Theorem 11. Our proof is based upon the reasoning of Maksimova (1986), but given in terms of the machinery described above. We moreover employ a characterisation of Levin (1969) as described by Wojtylak (2004). The refutation system we eventually obtain in Theorem 6 is the same as given by Skura (1992, Theorem 6.1).

11 Definition (Medvedev's Logic)

Per set $X$ the Medvedev frame, denoted $\mathrm{B}(X)$, is $\mathbf{P} X-\emptyset$ ordered by $\supseteq$. Medvedev's Logic ML is the intermediate logic defined by

$$
\mathrm{ML}:=\bigcap \operatorname{Th}(\{\mathrm{B}(X) \mid X \text { a finite set }\})
$$


Through the theorem of Glivenko (1929), we know that $\vdash_{\text {IPC }} \neg \phi$ holds if and only if $\vdash_{\text {CPC }} \neg \phi$ for any formula $\phi$. The equivalence below holds for any intermediate logic $L$ with the disjunction property, and for any finite set of formulae $\Delta$.

$$
\vdash_{\mathrm{L}} \bigvee_{\chi \in \Delta} \neg \chi \text { if and only if } \vdash_{\mathrm{CPC}} \neg \chi \text { for some } \chi \in \Delta \text {. }
$$

A formula is said to be disjunctive-negative in $L$ whenever, within $L$, it is equivalent to a formula of the above left-hand form. Note that this differs from the definition on Maksimova (1986), who requires a formula to be syntactically of the above form. We say that a formula $\phi$ is essentially negative when each variable occurs within the scope of a negation. More symbolically, the set of essentially negative formulae on $X$, denoted $\mathcal{L}_{-}(X)$, is defined by

$$
\mathcal{L}_{-}(X)::=\top|\perp| \neg \mathcal{L}(X)\left|\mathcal{L}_{-}(X) \wedge \mathcal{L}_{-}(X)\right| \mathcal{L}_{-}(X) \vee \mathcal{L}_{-}(X) \mid \mathcal{L}_{-}(X) \rightarrow \mathcal{L}_{-}(X) .
$$

A substitution $\sigma: \mathcal{L}(X) \rightarrow \mathcal{L}(Y)$ is said to be essentially negative if its image is contained in $\mathcal{L}_{-}(Y)$. Consider the intermediate logic ND defined below, as introduced by Maksimova (1986). It is a straightforward observation that the logic KP of Kreisel and Putnam (1957) is an extension of ND.

$$
\begin{aligned}
& \mathrm{ND}:=\mathrm{IPC}+\left\{\left(\neg x \rightarrow \bigvee_{i=1}^{n} \neg z_{i}\right) \rightarrow \bigvee_{i=1}^{n} \neg x \rightarrow \neg z_{i} \mid n \in \mathbb{N}\right\} \\
& \mathrm{KP}:=\mathrm{IPC}+\left(\neg x \rightarrow z_{1} \vee z_{2}\right) \rightarrow\left(\neg x \rightarrow z_{1}\right) \vee\left(\neg x \rightarrow z_{2}\right)
\end{aligned}
$$

We mention some elementary properties of KP and ML in Lemma 11, both of which are crucial for Theorem 4.

11 Lemma

The logics ML and KP are extensions of ND and both have the disjunction property.

Proof That KP has the disjunction property is proven by Kreisel and Putnam (1957, p. 75). By Maksimova (1986, Proposition 2) it is clear that ML has the disjunction property as well.

From the definitions of both KP and ND, it is immediate that the latter contains the former. Let us prove that $\mathrm{ML}$ is an extension of ND. To this end, let $X \neq \emptyset$ be a finite set and consider any model $v: \mathrm{B}(X) \rightarrow \mathbf{P} Y$. Let $W \subseteq \max \mathrm{B}(X)$ and $a \in \mathrm{B}(X)$ be such that $W \subseteq \max a \uparrow$. By Lemma 6, it suffices to show that there exists an element $b \in \mathrm{B}(X)$ satisfying both $a \leq b$ and $\max b \uparrow=W$. Such an element always exists, simply take $b:=\bigcup W$, and see that it satisfies all requirements.

In ND, all essentially negative formulae are in fact disjunctive negative, as we prove below. This statement is but a minor reformulation of Maksimova (1986, Lemma 4).

12 Lemma

Let $\phi \in \mathcal{L}(X)$ be a formula, and let $\sigma: \mathcal{L}(X) \rightarrow \mathcal{L}(Y)$ be an essentially negative substitution. There is a finite $\Delta \subseteq \mathcal{L}(Y)$ such that

$$
\vdash_{\mathrm{ND}} \sigma(\phi) \equiv \bigvee_{\chi \in \Delta} \neg \Delta .
$$


Moreover, $\vdash_{\mathrm{KP}} \phi$ precisely if $\vdash_{\mathrm{ML}} \phi .^{11}$

Proof The former statement can be proven by means of a straightforward inductive argument along the structure of $\phi$. Let us focus on the second statement, and prove the implication from left to right. Suppose that $\vdash_{K P} \phi$. Through (8) and Lemma 11 it follows that there is some $\chi \in \Delta$ such that $\vdash_{\mathrm{KP}} \neg \chi$. By Glivenko's theorem we now know that $\vdash_{\mathrm{ML}} \neg \chi$. Another application of (8) and Lemma 11 yield $\vdash_{\mathrm{ML}} \phi$ as desired.

Fix a finite maximally separable partial order $K$, and endow it with the valuation $\mathrm{m}_{K}$. From Lemma 9 we know that for all $a, b \in K$ the following holds.

$$
a \leq b \text { iff } b \Vdash \neg \bigvee(\max K-a \uparrow)
$$

Intuitively, this amounts to saying that $a$ lies below $b$ precisely when every maximal element that is not above $a$ is not above $b$. In maximally separable models, this naturally holds, as proven in Lemma 9. Through this equivalence, one can readily infer that the substitution $\rho$, as defined below, is such that $K \Vdash \rho(\phi) \equiv \phi$ for all formulae $\phi$.

$$
\rho: \mathcal{L}(\max K) \rightarrow \mathcal{L}(\max K), \quad m \mapsto \neg \bigvee(\max K-\{m\})
$$

We employ this substitution in Theorem 4 below. This theorem shows that to each formula that is non-derivability in $M L$ there must be a substitution instance that is not derivable in KP. We spell this out in a bit more detail in Corollary 4.

4 Theorem (Levin, 1969)

The following are equivalent for any formula $\phi \in \mathcal{L}(X)$ :

$1 \vdash_{\mathrm{ML}} \phi$;

2 For all finite sets $Y$ and all essentially negative substitutions $\sigma: \mathcal{L}(X) \rightarrow \mathcal{L}(Y)$ we have $\vdash_{\mathrm{KP}} \sigma(\phi)$.

Proof Suppose (1) holds and let $\sigma$ be essentially negative. From Lemma 12 we know that $\vdash_{\mathrm{ML}} \sigma(\phi)$ and $\vdash_{\mathrm{KP}} \sigma(\phi)$ are equivalent. Structurality ensures the former, hence (2) holds.

Now suppose that (1) does not hold. This gives us some finite $Z \neq \emptyset$ and a valuation $v: \mathrm{B}(Z) \rightarrow$ $\mathcal{L}(X)$ such that $v \| \phi$. The partial order $K:=\mathrm{B}(Z)$ is maximally separable, and hence the model $\mathrm{m}_{K}: K \rightarrow \mathcal{L}(\max K)$ is concrete due to Lemma 9. Indeed, we know that $k \in \mathrm{B}(X)$ is maximal precisely if $k$ is a singleton. Now observe that $a \leq\{x\}$ implies $b \leq\{x\}$ for all $x \in X$ precisely means that $a \subseteq b$, which is equivalent to $b \leq a$. This proves that $K$ is indeed maximally separable.

For convenience we write $Y:=\max K$. By Lemma 3, we know of a substitution $\tau: \mathcal{L}(X) \rightarrow$ $\mathcal{L}(Y)$ such that $\mathrm{m}_{K} \Vdash \tau(\psi)$ holds precisely if $v \Vdash \psi$. Now consider $\rho: \mathcal{L}(Y) \rightarrow \mathcal{L}(Y)$, as given in (9), and define $\sigma:=\rho \tau: \mathcal{L}(X) \rightarrow \mathcal{L}(\max K)$. It is clear that this substitution is essentially negative.

\footnotetext{
${ }^{11}$ Let us remark that through Maksimova (1986, Proposition 6) we might also replace this last statement with: for all intermediate logics $L$ above ND with the disjunction property one has that $\vdash_{\mathrm{L}} \phi$ holds precisely if $\vdash_{\mathrm{ND}} \phi$. The proof is analogous, and the original statement may be retrieved through Lemma 11.
} 
By Lemma 12, we now know that $\vdash_{\mathrm{KP}} \rho(\phi)$ entails $\vdash_{\mathrm{ML}} \rho(\phi)$. But then $\mathrm{m}_{K} \Vdash \rho(\phi)$, which ensures $\mathrm{m}_{K} \Vdash \tau(\phi)$ due to the remark above this theorem. This, in turn, would yield $v \Vdash \phi$, a contradiction proving (2) not to hold.

\section{Corollary}

Let $\vdash$ be the least structural consequence relation extending both $\vdash_{\mathrm{KP}}$ and $x \vee y /\{x, y\}$. For any formula $\phi$, we have that $\forall \mathrm{ML} \phi$ precisely if there is a substitution $\sigma$ and a finite set of formulae $\Delta$, each non-derivable in CPC, such that $\sigma(\phi) \vdash \Delta$.

Proof From left to right we assume $\nvdash_{\mathrm{ML}} \phi$. By Theorem 4, we get an essentially negative substitution $\sigma$ such that $\forall$ KP $\sigma(\phi)$. Due to Lemma 12, this gives to a set formulae $\Delta$ such that $\sigma(\phi) \vdash_{\mathrm{ND}} \bigvee \Delta$. Note that (7) ensures that each of the formulae in $\Delta$ is non-derivable in CPC. It follows from Lemma 11 that $\vdash_{\mathrm{ND}} \subseteq \vdash_{\mathrm{KP}}$, so the above combine to show $\sigma(\phi) \vdash \Delta$, as desired.

To prove the converse, we suppose $\sigma(\phi) \vdash \Delta$ for a particular substitution $\sigma$ and a set of formulae $\Delta$ non-derivable in CPC. It follows from Lemma 11 that $\vdash \subseteq \sim_{\mathrm{ML}}$. Now see that if $\vdash_{\mathrm{ML}} \phi$, then $\vdash_{\mathrm{ML}} \sigma(\phi)$ by structurality, so $\vdash_{\mathrm{ML}} \chi$ holds for some $\chi \in \Delta$. Yet this entails $\vdash_{\mathrm{CPC}} \chi$, a contradiction.

The following property is a generalisation of the above Corollary 4. In Theorem 5, we show that if a logic $L$ is such that $\vdash_{L}$ has this property then it is complete with respect to the refutation system generated by the set of non-derivable formulae $\Theta$ and rules $\mathcal{R}$. More concretely, in Theorem 6 we use this general machinery to give a refutation system for ML.

12 Definition (Admissibly Reducible)

We say that a consequence relation $\vdash$ is admissibly reducible to $\Theta$ through $\mathcal{R}$ when the following hold:

$1 \Theta$ is a set of formulae, and each formula in $\Theta$ is non-derivable in $\vdash$;

$2 \mathcal{R}$ is a set of rules closed under substitutions, and each rule in $\mathcal{R}$ is admissible with respect to $\vdash$;

3 for each $\phi$ the following equivalence holds

$$
\forall \mathrm{L} \phi \text { iff there is a } \sigma \text { and } \Delta \subseteq \Theta \text { such that } \sigma(\phi) \mathcal{R} \Delta \text {. }
$$

Note that, in the above definition, the formulae $\Theta$ are necessarily non-derivable in $L$, even without assuming this, whenever $\mathcal{R}$ contains reflexivity. Indeed, for each $\theta \in \Theta$ we then have that $\theta \mathcal{R}\{\theta\}$ and so $\forall \mathrm{L} \theta$ follows. However, it need not be the case that the formulae in $\Theta$ are non-derivable in CPC. ${ }^{12}$

Through a slight abuse of language, we will say that an intermediate logic $\mathrm{L}$ is admissibly reducible to $\Theta$ through $\mathcal{R}$ whenever $\vdash_{\mathrm{L}}$ is. Let us give the following lemma to illustrate the definition.

\footnotetext{
${ }^{12}$ Examples of this can be seen in Skura (1992). For instance, his Lemma 3.3 shows that Smetanich's logic (there called Heyting-Łukasiewicz logic) is admissibly reducible to $x \vee \neg x$ through $\vdash_{\text {IPC. }}$ We do not explore such logics in the following.
} 


\section{Lemma}

Let $L$ be an intermediate logic. Now $L$ is admissibly reducible to $\emptyset$ through $\sim$ precisely if $L=C P C$.

Proof From right to left, we assume $\phi \in \mathcal{L}(X)$ to be such that $\nvdash_{\mathrm{CPC}} \phi$. This gives a model $v:\{*\} \rightarrow \mathbf{P} X$ such that $v \forall \phi$, where $\{*\}$ is the one-point partial order. Define the substitution $\sigma: \mathcal{L}(X) \rightarrow \mathcal{L}(\emptyset)$ by mapping $x \in X$ to $\top$ if $x \in v(*)$ and to $\perp$ otherwise. It is easy to verify that $\sigma(\phi) \equiv \perp$, and thus $\sigma(\phi) \sim_{\mathrm{CPC}} \emptyset$.

To prove the other direction, we proceed by contraposition. Assume that $\mathrm{L} \subset \mathrm{CPC}$ and remark that $\phi:=\neg \neg x \rightarrow x$ is such that $\forall \mathrm{L} \phi$. Consider any substitution $\sigma$ and observe that CPC $\vdash \sigma(\phi)$. This yields a a substitution $\tau$ such that IPC $\vdash \tau(\sigma(\phi)) \equiv \top$. As a consequence, $\sigma(\phi) \vdash_{\mathrm{L}} \emptyset$ can not hold, proving the desired.

To connect Definition 12 to the above discussion, remark that in Corollary 4 it is argued that ML is admissibly reducible to the set of all non-derivable formulae in CPC through the set of rules

$$
\vdash_{\mathrm{KP}} \cup\{x \vee y /\{x, y\}\}
$$

\section{Theorem}

Let $L$ be an intermediate logic admissibly reducible to $\Theta$ through $\mathcal{R}$. The refutation system determined by $\Theta$ and $\mathcal{R}$ is complete and, hence $L$ is $E$-decidable. Moreover, when $\Theta$ is the set of formulae non-derivable in CPC then the refutation system determined by $\{\perp\}$ and $\mathcal{R} \cup \vdash_{\text {IPC }}$ is already complete.

Proof In order to prove completeness, we assume $\phi$ to be some formula such that $\forall \phi$. By assumption, we obtain a substitution $\sigma$ and a finite subset $\Delta \subseteq \Theta$ such that $\sigma(\phi) \mathcal{R} \Delta$. We now compute the following, where the first and last relation follow immediately by Definition 4 .

$$
\emptyset \vdash_{\mathrm{Rf}} \Delta \vdash_{\mathrm{Rf}} \sigma(\phi) \vdash_{\mathrm{Rf}} \phi
$$

Through transitivity, we thus infer $\vdash_{\mathrm{Rf}} \phi$.

Let us now focus on the final statement. To prove completeness, it suffices to show that $\perp \vdash_{\mathrm{Rf}} \theta$ for arbitrary $\theta \in \Theta$. Take $X$ such that $\theta \in \mathcal{L}(X)$ and know that, as $\forall$ CPC $\theta$ by assumption, there must be some $\sigma: \mathcal{L}(X) \rightarrow \mathcal{L}(\emptyset)$ such that $\vdash_{\mathrm{IPC}} \sigma(\theta) \equiv \perp$. This entails $\perp \vdash_{\mathrm{Rf}} \sigma(\theta) \vdash_{\mathrm{Rf}} \theta$, hence the desired follows.

6 Theorem (Skura, 1992)

The refutation system below is both sound and complete for ML.

$$
\frac{\dashv \perp}{\dashv} \mathrm{Ax} \quad \frac{\dashv \sigma(\phi)}{\dashv \phi} \text { Subs } \frac{\dashv \psi \quad \phi \vdash_{\mathrm{KP}} \psi}{\dashv \phi} \operatorname{Inv} \vdash_{\mathrm{KP}} \quad \frac{\dashv \phi \quad \dashv \psi}{\dashv \phi \vee \psi} \mathrm{RDP}
$$

Proof This is a direct consequence of Lemma 2, Theorem 5 and Corollary 4.

The remainder of this section is devoted to developing the conditions under which not-down formulae of models can play the role of $\phi$ in Definition 12. Let us take a brief detour through admissible approximations. Recall the motivation of this notion, as given at the start of this section. To be 
concrete, we say that $\psi$ is an admissible approximation of $\phi$ whenever the following holds for all formulae $\chi$ :

$$
\phi \vdash \chi \text { if and only if } \psi \vdash \chi \text {. }
$$

The intuitive feel to the following is that one can iterative compute the admissible approximation of a formula nd $\mathrm{m}_{K}$, whenever $K$ is maximally distinguishable and proper, so in particular when $K$ is maximally separable. One can think of this iteration as traversing the tree from the root to all of its leaves. At each stage, one considers a set of nodes and their corresponding not-downset formulae. Step-by-step we replace a node by its immediate successors, if it has any. Throughout this process, the admissible approximation of the disjunction of these formulae remains invariant.

In Lemma 14, we show sufficient conditions for maintaining this invariant. Theorem 8 provides the evidence needed to show that when the computation ends one is left with an admissible approximation. Finally, all of this is put together in Theorem 9. It might be helpful to remark that Lemma 14 and Theorem 9 play roles similar to those of Lemma 17 and Theorem 3 of Goudsmit and Iemhoff (2014).

We should note that, in order to apply Theorem 5, our eventual end-goal, it will not be necessary to know that this computation actually delivers an admissible approximation. Indeed, it suffices to merely know that $n d \mathrm{~m}_{K}$ can be admissibly transformed into a disjunctive-negative formula. We do prove this stronger fact to stress the connection between the proof technique as employed by Skura (1989, Theorem 2) and methods commonly used in the study of admissibility. In the following, we write $\vdash_{\mathrm{L}, \overline{\mathrm{D}}_{n}}$ to mean $\left(\vdash_{\mathrm{L}}\right)_{\overline{\mathrm{D}}_{n}}$.

\section{Lemma}

Let $n$ be a natural number, and let $\mathrm{L}$ be an intermediate logic with the finite model property. Take $W \subseteq \mathrm{U}_{\mathrm{L}}(X)$ to be an anti-chain with $1 \neq|W| \leq n$ such that $\operatorname{Th}(W) \cap X=\emptyset$, and let $k \in \mathrm{U}_{\mathrm{L}}(X)$ be such that $W \kappa k$. It follows that

$$
\text { nd } k \vdash_{\mathrm{L}, \overline{\mathrm{D}}_{n}}\{\text { nd } w \mid w \in W\} \text { and } \bigvee_{w \in W} \operatorname{nd} w \vdash_{\mathrm{L}} \text { nd } k \text {. }
$$

Proof Before we proceed, first recall that by Lemma 10 we have

$$
\vdash_{\mathrm{L}}\left(\mathrm{up} k \rightarrow \bigvee_{w \in W} \mathrm{nd} w\right) \equiv \mathrm{nd} k .
$$

The second statement trivially follows from this observation. We now focus on the former statement. Let us first apply the above equivalence and unfold the definition of up $(-)$.

$$
\vdash_{\mathrm{L}} \mathrm{nd} k \equiv\left(\operatorname{up} k \rightarrow \bigvee_{w \in W} \mathrm{nd} w\right) \equiv\left(\left(\bigvee_{w \in W} \mathrm{nd} w \rightarrow \bigvee_{w \in W} \mathrm{up} w\right) \rightarrow \bigvee_{w \in W} \mathrm{nd} w\right) .
$$


The desired now follows from the computations below.

$$
\begin{aligned}
\text { nd } k+\vdash_{\mathrm{L}} & \left(\bigvee_{w \in W} \mathrm{nd} w \rightarrow \bigvee_{w \in W} \text { up } w\right) \rightarrow \bigvee_{w \in W} \text { nd } w \\
\vdash_{\mathrm{L}, \overline{\mathrm{D}}_{n}} & \left\{\left(\bigvee_{w \in W} \mathrm{nd} w \rightarrow \bigvee_{w \in W} \text { up } w\right) \rightarrow \quad \text { nd } v \mid v \in W\right\} \\
\vdash_{\mathrm{L}} \quad\{\mathrm{nd} w \mid w \in W\} &
\end{aligned}
$$

The first line is due to the assumed equivalence and the second is an immediate application of $\mathrm{D}_{n}^{-}$. Finally, see that for each $v \in W$ we have

$$
v \Downarrow\left(\bigvee_{w \in W} \operatorname{nd} w \rightarrow \operatorname{up} W\right) \rightarrow \operatorname{nd} v .
$$

Through Corollary 1, we now know the last inference to be valid as well, which finishes the proof.

To prove Theorem 8 , we introduce some additional terminology and results from the literature. ${ }^{13}$ We say that a formula $\phi$ is L-projective whenever there is a substitution $\sigma$ such that $\vdash_{\mathrm{L}} \sigma(\phi)$ and for all formulae $\psi$ we have $\phi \vdash_{\mathbf{L}} \sigma(\psi) \equiv \psi$. The subsequent theorem is a reformulation of the original characterisation of projective formulae due to Ghilardi (1999), here given in the context of the universal model.

\section{Theorem (Ghilardi, 1999)}

Let $\mathrm{L}$ be an intermediate logic with the finite model property. A formula $\phi \in \mathcal{L}(X)$ is $\mathrm{L}$-projective precisely if there is a $k \in \mathrm{U}_{\mathrm{L}}(X)$ with $k \Vdash \phi$ and for all anti-chains $W \subseteq \mathrm{U}_{\mathrm{L}}(X)$ with $W \Vdash \phi$ we have a $l \Vdash \phi$ with $W \kappa l$ whenever there is a $k \in \mathrm{U}_{\mathrm{L}}(X)$ with $W \kappa k$.

\section{Theorem}

Let $\mathrm{L}$ be an intermediate logic with the finite model property, let $X$ be a finite set, and let $W \subseteq$ $\mathrm{U}_{\mathrm{L}}(X)$ be an upset. The following are equivalent.

1 The formula nd $k$ is projective, for all $k \in \mathrm{U}_{\mathrm{L}}(X)$ such that $W \kappa k$ and $k \notin W$;

2 There are distinct $k, k^{\prime} \in \mathrm{U}_{\mathrm{L}}(X)$ such that $W \kappa k, k^{\prime}$.

Proof Suppose (2) holds, and let $k \in W$ be such that $W \kappa k$. We proceed through Theorem 7. By assumption, we know of a $k^{\prime} \neq k$ such that $W \kappa k^{\prime}$. As a consequence, $k^{\prime} \not \leq k$, proving $k^{\prime} \Vdash$ nd $k$ by Theorem 2 .

Let $V \subseteq \mathrm{U}_{\mathrm{L}}(X)$ be such that $V \Vdash$ nd $k$, and assume that there is a $l \in \mathrm{U}_{\mathrm{L}}(X)$ with $V \kappa l$. If $l \in V$ then we are done, so assume the contrary. To prove the desired, we seek a $l^{\prime} \in \mathrm{U}_{\mathrm{L}}(X)$ with $l^{\prime} \Vdash$ nd $k$ and $V \kappa l$. If $l \not \leq k$ then simply pick $l=l^{\prime}$. Suppose $l \leq k$, then either $l=k$ or $l \in W \uparrow$. In the latter case, we know $w \leq l \leq k$ for some $w \in W$, so this case never occurs.

\footnotetext{
${ }^{13}$ Through Theorem 7 one can see that Bezhanishvili and de Jongh (2012, Theorem 4.13) is equivalent to Theorem 8.
} 
Assuming $k=l$ we compute

$$
k \uparrow=W \uparrow \cup\{k\}=W \cup\{k\}=V \cup\{l\}=V \uparrow \cup\{l\}=l \uparrow,
$$

and so $V=V-\{l\}=W-\{k\}=W$. By assumption, we know of a $k^{\prime} \neq k$ such that $W \kappa k^{\prime}$, hence $V \kappa k^{\prime}$. Now take $k^{\prime}=l^{\prime}$ and we have proven (1).

To prove the converse, we reason by contraposition, so assume the negation of (2). This means that $W$ can have either no covers at all, or a unique one. In the former case, the desired is vacuously true, so suppose there is but one unique $k \in \mathrm{U}_{\mathrm{L}}(X)$ such that $W \kappa k$. Clearly, $W \Vdash$ nd $k$ because if $w \in W$ were such that $w \|$ nd $k$ then $w \leq k$, and as $k \leq w$ this entails $k \in W$, a contradiction. But if $l \in \mathrm{U}_{\mathrm{L}}(X)$ is such that $W \kappa l$, then $l=k$ holds by assumption, hence $l \Vdash$ nd $k$ can never hold. Through Theorem 7, this proves the negation of (1).

Before we can state the following theorem we need two additional definitions.

13 Definition (Branching)

Let $K$ be a partial order. The branching degree of an element $k \in K$ is its number of immediate successors. We say $K$ is at most $n$-fold branching when the branching degree of all $k \in K$ is bounded by $n$.

In Theorem 9 below we prove that, for any finite rooted maximally separated models $K$, the admissible approximation of its not-down formula equals the disjunction of the not-down formulae of its maximal nodes. This can be readily seen when one instantiates $\Delta$ by nd $K$ and $\bigvee\{$ nd $m \mid m \in \max (K)\}$, making (10) and (11) true. Note that we take special care to keep track of the rules we use in this argument.

\section{Theorem}

Let $n$ be a natural number, and let $L$ be an intermediate logic with the finite model property that admits $\overline{\mathrm{D}}_{n}$. Let $K$ be a rooted finite maximally separable partial order, and endow it with the valuation $\mathrm{m}_{K}$. Assume that $K$ is at most $n$-fold branching. The following are equivalent, for all finite set of formulae formulae $\Delta$.

$$
\begin{array}{rr}
\operatorname{nd} K \vdash_{\mathrm{L}} & \Delta \\
\text { nd } K \vdash_{\mathrm{L}, \overline{\mathrm{D}}_{n}} & \Delta
\end{array}
$$

Proof We claim that the following holds for all $k \in K$.

$$
\text { nd } k \vdash_{\mathrm{L}, \overline{\mathrm{D}}_{n}}\{\text { nd } m \mid m \in \max (k \uparrow)\} \text { and } \bigvee\{\text { nd } m \mid m \in \max (k \uparrow)\} \vdash_{\mathrm{L}} \text { nd } k \text {. }
$$

Assume that the claim holds. It is easy to see that (12) entails (10), because $\vdash_{L, \bar{D}_{n}}$ is contained in $r_{L}$. We also note that (11) and the first conjunct of (13) immediately yield (12).

Now suppose that (10) holds. The second conjunct of (13) leads to the following, bearing in mind that $\vdash_{\mathrm{L}, \overline{\mathrm{D}}_{n}} \subseteq r_{\mathrm{L}}$.

$$
\bigvee\{\text { nd } m \mid m \in \max (K)\} \sim_{\mathrm{L}} \Delta
$$


Due to Theorem 8, we know that for each $m \in \max (K)$ the formula nd $m$ is L-projective. Fix a $m \in \max (K)$ and know that we have, due to the projectivity of nd $m$, a substitution $\sigma_{m}$ such that $\vdash_{\mathrm{L}} \sigma_{m}($ nd $m)$ and nd $m \vdash_{\mathrm{L}} \sigma_{m}(\phi) \equiv \phi$ for all $\phi$. As $\vdash_{\mathrm{L}} \sigma_{m}$ (nd $\left.m\right)$ holds, we know of some $\chi \in \Delta$ such that $\vdash_{\mathrm{L}} \sigma_{m}(\chi)$ by the above. By the second property of $\sigma_{m}$, it now follows that nd $m \vdash_{\mathrm{L}} \chi$. Through transitivity, we thus obtain the following.

$$
\bigvee\{\text { nd } m \mid m \in \max (K)\} \vdash_{\mathrm{L}} \Delta
$$

Combining this with the first conjunct of (10) we get (11), as desired.

Let us now prove the claim (13) by well-founded induction along the order in $K$ for all $k \in K$. Write $W$ for the set of immediate successors of $k \in K$. If $W$ is empty, then there is nothing left to prove, so assume the contrary.

We compute as below. The first line makes use of Lemma 14, and the second holds by induction and transitivity. The third line follows mainly from transitivity of consequence relations, keeping in mind that $\max (k \uparrow)=\bigcup_{w \in W} \max (w \uparrow)$. The subsequent three lines follow for analogous reasons in the reversed order.

$$
\begin{aligned}
& \operatorname{nd} k \quad \vdash_{\mathrm{L}, \overline{\mathrm{D}}_{n}} \quad\{\mathrm{nd} w \mid w \in W\} \\
& \vdash_{\mathrm{L}, \overline{\mathrm{D}}_{n}} \bigcup_{w \in W}\{\mathrm{nd} m \mid m \in \max (w \uparrow)\} \\
& \vdash_{\overline{\mathrm{D}}_{n}} \quad\{\mathrm{nd} m \mid m \in \max (k \uparrow)\} \\
& \bigvee\{\operatorname{nd} m \mid m \in \max (k \uparrow)\} \quad \vdash_{\mathrm{L}} \quad \bigvee_{w \in W} \bigvee\{\text { nd } m \mid m \in \max (w \uparrow)\} \\
& \vdash_{\mathrm{L}} \quad \bigvee_{w \in W} \mathrm{nd} w \\
& \vdash_{\mathrm{L}} \quad \text { nd } k
\end{aligned}
$$

\section{Refutation for $T_{n}$}

Gabbay and de Jongh (1974) introduced an infinite series of finitely axiomatizable intermediate logics with both the disjunction property and finite model property, now known as the Gabbay-de Jongh logics, given in Definition 14 below. ${ }^{14}$ Per natural number $n$, one can consider the intermediate logic complete with respect to finite trees with branching of degree at most $n$. This is the $(n-1)^{\text {th }}$ Gabbay-de Jongh logic. Chagrov and Zakharyaschev (1997) call this logic $\mathbf{T}_{n}$, and we will refer to it as such from now on. An axiomatization of this logic is given below, immediately followed by a characterization of the concrete models of this logic.

14 Definition

The logic of bounded branching until the $n^{\text {th }}$ degree, $T_{n}$, is given as the intermediate logic

$$
\mathrm{T}_{n}:=\mathrm{IPC}+\bigwedge_{i=1}^{n+2}\left(\left(x_{i} \rightarrow \bigvee_{j \neq i} x_{j}\right) \rightarrow \bigvee_{j \neq i} x_{j}\right) \rightarrow \bigvee_{i=1}^{n+2} x_{i}
$$

\footnotetext{
${ }^{14}$ As attested to by Segerberg (1973), this result was already known and published in a technical report in 1969.
} 
The following series of results are meant to describe the structure of concrete models of $T_{n}$. In Lemma 15 , we, roughly speaking, describe models of $T_{n}$ as those models where only anti-chains of size at most $n$ can cover an element.

15 Lemma (Goudsmit, 2013b, Lemma 12)

Let $K$ be a concrete and image-finite model $K$ model. Now $K$ is a model of $\mathrm{T}_{n}$ precisely if for each anti-chain $W \subseteq K$ and each $k \in K$ we have $|W| \leq n$ whenever $W \kappa k$.

5 Corollary

A concrete and image-finite model whose underlying partial order is a tree is a model of $\mathrm{T}_{n}$ precisely if it is at most $n$-fold branching.

6 Corollary

For each set of variables $X$ the universal model $\mathrm{U}_{\mathrm{T}_{n}}(X)$ is strongly $n$-ary covered. In particular, $\overline{\mathrm{D}}_{m}$ is admissible for each $m \leq n$.

Proof The first statement is immediate from the observation that every anti-chain in the universal model covers something, and Lemma 15. The second statement follows from the first through Corollary 2.

The remainder of this section will be used to argue that the refutation system as given in Theorem 11 is both sound and complete. The system given there is the refutation system determined by $\{\perp\}$ and $\bar{D}_{n}$. We prove sound- and completeness by means of the machinery developed in Section 3. In short, we will show that $\mathrm{T}_{n}$ admissibly reduces to the set of CPC non-derivable formulae through $\overline{\mathrm{D}}_{n}$.

16 Lemma

For each natural $n \geq 2$, the logic $\mathrm{T}_{n}$ is sound and complete with respect to proper at most $n$-fold branching finite trees. Moreover, each of these trees is maximally separable.

Proof Soundness is immediate through Corollary 5. Let us now argue for completeness. Take $\phi$ to be a formula such that $\mathrm{T}_{n} \nvdash \phi \phi$. By Gabbay and de Jongh (1974, Equation 34) we obtain a finite and at most $n$-fold branching tree such that $T \forall \phi$. Without loss of generality we may assume that $T$ is indeed proper, taking care to note that $n \geq 2$. The final statement is immediate from Lemma 8.

10 Theorem

For any $n \geq 2$, the intermediate logic $\mathrm{T}_{n}$ is admissibly reducible to the set formulae that are non-derivable in CPC through all instances of $\overline{\mathrm{D}}_{n}$ and derivability in IPC.

Proof Suppose that $\phi$ is such that $\forall_{\mathrm{T}_{n}} \phi$. Then there is a maximally separable model $K \Vdash \mathrm{T}_{n}$ of branching degree at most $n$ such that $K \forall \phi$ by Lemma 16. From Lemma 5 we get a substitution such that $\sigma(\phi) \vdash_{\text {IPC }}$ nd $K$. Through Theorem 9 we know the following.

$$
\text { nd } K \vdash_{\mathrm{IPC}_{\overline{\mathrm{D}}} \overline{\mathrm{D}}_{n}} \bigvee\{\text { nd } m \mid m \in \max (K)\}
$$

We combine the above two observations into:

$$
\sigma(\phi) \vdash_{\mathrm{IPC}} \mathrm{nd} K \vdash_{\mathrm{IPC}, \overline{\mathrm{D}}_{n}} \bigvee\{\mathrm{nd} m \mid m \in \max (K)\} .
$$

Finally, we remark that $\vdash_{\mathrm{IPC}, \overline{\mathrm{D}}_{n}}$ is included in $\sim_{\mathrm{T}_{n}}$ via Corollary 6. 
The following two theorems are our main results. Note that, due to Remark 1, we may replace $\mathrm{R} \overline{\mathrm{D}}_{n}$ with the basis of $\mathrm{T}_{n}$ as described by Goudsmit and Iemhoff (2014). This too would yield a structural refutation system, yet its formulation would be more cumbersome. Theorem 12 is to $\mathrm{T}_{n}$ what Iemhoff (2001a, Theorem 3.5) is to IPC, and Maksimova (1986, Theorem 5) is to ML, namely, a characterisation of an intermediate logic as the strongest intermediate logic that admits a particular set of rules.

11 Theorem

For any $n \geq 2$ the refutation system below is sound and complete for the intermediate logic $T_{n}$. In particular, $T_{n}$ is $€$-decidable.

$$
\begin{gathered}
\frac{}{\dashv \perp} \operatorname{Ax} \quad \frac{\dashv \sigma(\phi)}{\dashv \phi} \text { Subs } \frac{\dashv \psi}{\dashv \phi} \quad \frac{\phi \vdash_{\mathrm{IPC}} \psi}{\mathrm{HT}} \mathrm{MT} \\
\frac{\dashv\left(\bigvee_{i=1}^{n} \chi_{i} \rightarrow \phi\right) \rightarrow \chi_{j} \text { per } j=1, \ldots, n}{\dashv\left(\bigvee_{i=1}^{n} \chi_{i} \rightarrow \phi\right) \rightarrow \bigvee_{j=1}^{n} \chi_{j}} \mathrm{RD}_{n}
\end{gathered}
$$

Proof This is a consequence of Theorem 10, Theorem 5, and Lemma 2, bearing Remark 1 in mind.

12 Theorem

For any $n \geq 2$ the intermediate logic $T_{n}$ is the greatest intermediate logic that admits $\bar{D}_{n}$.

Proof Let $L$ be an intermediate logic that is greater than $T_{n}$ which admits $\bar{D}_{n}$. There must be some $\phi$ such that $\mathrm{L} \vdash \phi$ yet $\mathrm{T}_{n} \not \forall \phi$. By Theorem 11 we know $\dashv \phi$. Now remark that this refutation system is sound for $\mathrm{L}$ too, yielding $\nvdash \mathrm{L} \phi$, a contradiction.

\section{Acknowledgements}

Support by the Netherlands Organisation for Scientific Research under grant 639.032 .918 is gratefully acknowledged. I would like to thank Tomasz Skura for sending me his paper "A complete syntactical characterization of the intuitionistic logic". During my visit to Bern I discussed this paper with George Metcalfe, which helped me understand its importance. Wojciech Dzik pointed me to the literature on refutation systems. When I presented an early version of this work at TACL 2013 Alex Citkin gave me wonderful feedback and further references, for which I am immensely grateful. I also would like to thank Nick Bezhanishvili and Rosalie Iemhoff for the many conversations on this work. Finally, I would like to thank the anonymous referee for their insightful comments, which helped to improve this paper.

\section{References}

Bezhanishvili, N. (2004). "De Jongh's characterization of intuitionistic propositional calculus". In: Liber Amicorum Dick de fongh. Ed. by J. van Benthem et al. University of Amsterdam.

- (2006). "Lattices of intermediate and cylindric modal logics". PhD thesis. Amsterdam University. ISBN: 9057761475. 
Bezhanishvili, N. and D. H. J. de Jongh (2012). "Extendible Formulas in Two Variables in Intuitionistic Logic”. In: Studia Logica 100.1-2 (1), pp. 61-89. ISSN: 0039-3215. DOI: 10.1007/s11225-0129389-8.

Chagrov, A. and M. Zakharyaschev (1991). "The Disjunction Property of Intermediate Propositional Logics”. In: Studia Logica 50.2, pp. 189-216. ISSN: 00393215. DOI: 10.1007/BF00370182.

- (1997). Modal Logic. Vol. 77. Oxford Logic Guides. Oxford University Press.

Cintula, P. and G. Metcalfe (2010). "Admissible rules in the implication-negation fragment of intuitionistic logic". In: Annals of Pure and Applied Logic 162.2, pp. 162-171. IssN: 0168-0072. DoI: 10.1016/j.apal.2010.09.001.

Citkin, A. (1977). "On Admissible Rules of Intuitionistic Propositional Logic". In: Mathematics of the USSR-Sbornik 31.2, pp. 279-288. DOI: 10.1070/SM1977v031n02ABEH002303.

- (1979a). “О Модальной Логике Интуиционистской Допустимости”. Russian. In: Modal and Tense Logic, Second Soviet-Finnish Colloquium in Logic. English translation of title: On modal logic of intuitionistic admissibility. Moscow, pp. 105-107.

- (1979b). “О Проверке Допустимости Не́которых Правил Интуиционистской Логике”. Russian. In: V-th All-Union Conference in Mathematical Logic. English translation of title: On verification of admissibility of some rules of intuitionistic logic. Novosibirsk, p. 162.

- (2008). "A mind of a non-countable set of ideas". In: Logic and Logical Philosophy 17.1-2. ISsN: 2300-9802. DOI: 10.12775/LLP.2008.003.

- (2012). "A note on admissible rules and the disjunction property in intermediate logics". In: Archive for Mathematical Logic 51.1-2 (1), pp. 1-14. IsSN: 0933-5846. DOI: 10.1007/s00153-0110250-y.

de Jongh, D. H. J. (1968). "Investigations on the Intuitionistic Propositional Calculus". PhD thesis. University of Wisconsin.

Dutkiewicz, R. (1989). "The method of axiomatic rejection for the intuitionistic propositional logic". In: Studia Logica 48.4, pp. 449-459. ISSN: 0039-3215. DoI: 10.1007/BF00370199.

Friedman, H. (1975). "One Hundred and Two Problems in Mathematical Logic". In: The fournal of Symbolic Logic 40.2, pp. 113-129. ISSN: 00224812. DoI: 10.2307/2271891.

Gabbay, D. M. and D. H. J. de Jongh (1974). "A Sequence of Decidable Finitely Axiomatizable Intermediate Logics with the Disjunction Property”. In: The fournal of Symbolic Logic 39.1, pp. 67-78. ISSN: 00224812. DOI: $10.2307 / 2272344$.

Ghilardi, S. (1999). "Unification in Intuitionistic Logic”. In: The fournal of Symbolic Logic 64.2, pp. 859-880. ISSN: 00224812. DOI: $10.2307 / 2586506$.

Glivenko, V. (1929). "Sur quelques points de la logique de M. Brouwer". In: Bulletin de la Classe des Sciences de l’Académie Royale de Belgique 15.5, pp. 183-188.

Gödel, K. (1932). "Zum intuitionistischen Aussagenkalkül”. In: Akademie der Wissenschaftischen in Wien, Mathematisch-naturwissenschaftliche Klasse, Anzeiger 69, pp. 65-66.

Goranko, V. (1994). "Refutation Systems in Modal Logic". In: Studia Logica 53.2, pp. 299-324. IssN: 00393215. DOI: $10.1007 / \mathrm{BF} 01054714$.

Goudsmit, J. P. (2013a). “A Note on Extensions: Admissible Rules via Semantics”. In: Logical Foundations of Computer Science. Ed. by S. Artemov and A. Nerode. Vol. 7734. Lecture Notes in Computer Science. Springer Berlin Heidelberg, pp. 206-218. ISBN: 978-3-642-35721-3. DOI: 10.1007/978-3642-35722-0_15.

- (2013b). "The Admissible Rules of $\mathrm{BD}_{2}$ and GSc". In: Logic Group Preprint Series 313. To appear in the Notre Dame Journal of Formal Logic, pp. 1-24. 
Goudsmit, J. P. and R. Iemhoff (2014). "On unification and admissible rules in Gabbay-de Jongh logics”. In: Annals of Pure and Applied Logic 165.2, pp. 652-672. ISSN: 0168-0072. DOI: 10.1016/j. apal.2013.09.003.

Grigolia, R. (1995). "Free and projective Heyting and monadic Heyting algebras". In: Non-Classical Logics and their Applications to Fuzzy Subsets. Ed. by U. Höhle and E. Klement. Vol. 32. Theory and Decision Library. Springer Netherlands, pp. 33-52. IsBN: 978-94-010-4096-9. DOI: 10.1007/97894-011-0215-5_4.

Harrop, R. (1960). "Concerning Formulas of the Types $A \rightarrow A \vee B, A \rightarrow(\exists x) B(x)$ in Intuitionistic Formal Systems”. In: The fournal of Symbolic Logic 25.1, pp. 27-32. IsSN: 00224812. DOI: 10.2307/ 2964334.

Iemhoff, R. (2001a). "A(nother) characterization of intuitionistic propositional logic". In: Annals of Pure and Applied Logic 113.1-3. First St. Petersburg Conference on Days of Logic and Computability, pp. 161-173. ISSN: 0168-0072. DOI: 10.1016/S0168-0072(01)00056-2.

- (2001b). "On the Admissible Rules of Intuitionistic Propositional Logic". In: The fournal of Symbolic Logic 66.1, pp. 281-294. ISSN: 00224812. DOI: 10.2307/2694922.

- (2005). "Intermediate Logics and Visser's Rules". In: Notre Dame fournal of Formal Logic 46.1, pp. 65-81. DoI: 10.1305/ndjfl/1107220674.

- (2013). “A Note on Consequence Relations". In: Logic Group Preprint Series 314.

Jankov, V. (1963). "Some superconstructive propositional calculi”. In: Soviet Mathematics Doklady 4. Translation of Doklady Akademii Nauk SSSR 151, 796-798 (1963), pp. 1103-1105. ISSN: 0197-6788.

Jeřábek, E. (2005). “Admissible Rules of Modal Logics”. In: Journal of Logic and Computation 15.4, pp. 411-431. DoI: 10.1093/logcom/exi029.

- (2010). "Bases of Admissible Rules of Łukasiewicz Logic". In: Journal of Logic and Computation 20.6, pp. 1149-1163. DOI: 10.1093/logcom/exp082.

Johansson, I. (1937). "Der Minimalkalkül, ein reduzierter intuitionistischer Formalismus". In: Compositio Mathematica 4, pp. 119-136.

Kleene, S. C. (1962). "Disjunction and Existence Under Implication in Elementary Intuitionistic Formalisms”. In: The fournal of Symbolic Logic 27.1, pp. 11-18. ISSN: 00224812. DOI: 10.2307/ 2963675.

Kracht, M. (1999). "Book Review: V. V. Rybakov. Admissibility of Logical Inference Rules”. In: Notre Dame fournal of Formal Logic 40.4, pp. 578-587. Dor: 10.1305/ndjfl/1012429722.

Kreisel, G. and H. W. Putnam (1957). "Eine Unableitbarkeitsbeweismethode für den Intuitionistischen Aussagenkalkül”. In: Archiv für mathematische Logik und Grundlagenforschung 3 (3-4), pp. 74-78. ISSN: 0003-9268. DOI: 10.1007/BF01988049.

Levin, L. A. (1969). "Some syntactical theorems on the Yu. T. Medvedev's calculus of finite problems”. In: Soviet Mathematics Doklady 10.2. Translation of Doklady Akademii Nauk SSSR 185, 32-33 (1969), pp. 288-289. ISSN: 0197-6788.

Lorenzen, P. (1955). Einführung in die operative Logik und Mathematik. Vol. 78. Die Grundlehren der mathematischen Wissenschaften in Einzeldarstellungen. Springer-Verlag.

Łukasiewicz, J. (1951). Artistotle's Syllogistic from the standpoint of modern formal logic. Oxford: Clarendon Press.

- (1952). "On the intuitionistic theory of deduction". In: Indagationes Mathematicae 14, pp. 202212.

Maksimova, L. L. (1986). “On Maximal Intermediate Logics with the Disjunction Property”. In: Studia Logica 45.1, pp. 69-75. ISSN: 00393215. DOI: 10.1007/BF01881550. 
Minari, P. and A. Wroński (1988). "The property (HD) in intermediate logics. A partial solution of a problem of H. Ono." In: Reports on Mathematical Logic 22, pp. 21-25.

Mints, G. E. (1976). "Derivability of admissible rules". In: fournal of Mathematical Sciences 6 (4). Translation from Zap. Nauchn. Semin. Leningr. Otd. Mat. Inst. Steklova 32, 85-89, pp. 417-421. ISSN: 1072-3374. DOI: $10.1007 / \mathrm{BF} 01084082$.

Prucnal, T. (1979). “On Two Problems of Harvey Friedman”. In: Studia Logica 38 (3), pp. 247-262. ISSN: 0039-3215. DOI: 10.1007/BF00405383.

Rozière, P. (1992). "Règles admissibles en calcul propositionnel intuitionniste". PhD thesis. Université de Paris VII.

Rybakov, V. V. (1984). "A criterion for admissibility of rules in the model system S4 and the intuitionistic logic”. In: Algebra and Logic 23 (5), pp. 369-384. ISSN: 0002-5232. DOI: 10.1007 / BF01982031.

Rybakov, V. V., M. Terziler, and Ç. Gencer (1999). "An Essay on Unification and Inference Rules for Modal Logics”. In: Bulletin of the Section of Logic 23.3, pp. 145-157.

Scott, D. (1957). "Completeness Proofs for the Intuitionistic Sentential Calculus". In: Summaries of talks presented at the summer institute for symbolic logic. Second Edition 25 July 1960. Communications Research Division, Institute for Defence Analyses, pp. 231-241.

- (1971). "On Engendering an Illusion of Understanding". In: The fournal of Philosophy 68.21. SixtyEighth Annual Meeting of the American Philosophical Association Eastern Division, pp. 787807. ISSN: 0022362X. DOI: $10.2307 / 2024952$.

- (1974). "Completeness and Axiomatizability in Many-Valued Logic". In: Proceedings of the Tarski Symposium. Vol. 25. Proceedings of Symposia in Pure Mathematics, pp. 411-435.

Segerberg, K. (1973). "Review: C. G. McKay, A Note on the Jaskowski Sequence”. In: The fournal of Symbolic Logic 38.3, pp. 520-521. ISsN: 00224812. DOI: 10.2307/2273058.

Skura, T. F. (1989). "A complete syntactical characterization of the intuitionistic logic". In: Reports on Mathematical Logic 23, pp. 75-80.

Skura, T. F. (1990). "A new criterion of decidability for intermediate logics". In: Bulletin of the Section of Logic 19.1, pp. 10-14.

- (1992). "Refutation Calculi for Certain Intermediate Propositional Logics". In: Notre Dame fournal of Formal Logic 33.4, pp. 552-560. DoI: 10.1305/ndjfl/1093634486.

- (1994). "Syntactic Refutations agains Finite Models in Modal Logic". In: Notre Dame fournal of Formal Logic 35.4, pp. 595-605. DOI: 10.1305/ndjfl/1040408615.

- (1999). Aspects of refutation procedures in the intuitionistic logic and related modal systems. Ed. by J. Hawranek. Vol. 15. Logika. Wydawnictwo Uniwersytetu Wrocławskiego. ISBN: 83-229-1985-9.

- (2005). "Intuitionistic Socratic procedures". In: fournal of Applied Non-Classical Logics 15.4, pp. 453464. DOI: $10.3166 /$ jancl.15.453-464.

- (2009). “A Refutation Theory”. In: Logica Universalis 3 (2), pp. 293-302. ISSN: 1661-8297. DOI: 10.1007/s11787-009-0009-y.

Słupecki, J. and G. Bryll (1973). "Proof of Ł-decidability of Lewis system S5”. In: Studia Logica 32.1, pp. 99-105. IsSN: 0039-3215. DOI: 10.1007/BF02123824.

Słupecki, J., G. Bryll, and U. Wybraniec-Skardowska (1971). "Theory of rejected propositions. I". In: Studia Logica 29.1, pp. 75-115. IsSN: 0039-3215. DOI: 10.1007/BF02121863.

- (1972). "The theory of rejected propositions. II". In: Studia Logica 30.1, pp. 97-139. IssN: 00393215. DOI: $10.1007 / \mathrm{BF} 02120839$. 
Smoryński, C. (1973). "Applications of Kripke models". In: Metamathematical Investigation of Intuitionistic Arithmetic and Analysis. Ed. by A. S. Troelstra. Vol. 344. Lecture Notes in Mathematics. Springer Berlin / Heidelberg, pp. 324-391. Dor: 10.1007/BFb0066744.

Surma, S. J., A. Wroński, and S. Zachorowski (1975). "On Jaśkowski-type semantics for the intuitionistic propositional logic”. In: Studia Logica 34.2, pp. 145-148. IssN: 0039-3215. DoI: 10.1007/ BF02123383.

Troelstra, A. S. and D. van Dalen (1988). "Logic". In: Constructivism in Mathematics - An Introduction. Vol. 121. Studies in Logic and the Foundations of Mathematics. Elsevier, pp. 35-111. Dor: 10.1016/ S0049-237X(09)70527-0.

Visser, A. (1984). "Evaluation, provably deductive equivalence in Heyting's arithmetic of substitution instances of propositional formulas". In: Logic Group Preprint Series 4.

- (2002). "Substitutions of $\Sigma_{0}^{1}$-sentences: explorations between intuitionistic propositional logic and intuitionistic arithmetic". In: Annals of Pure and Applied Logic 114.1-3, pp. 227-271. IssN: 0168-0072. DOI: 10.1016/S0168-0072(01)00081-1.

Wójcicki, R. (1988). Theory of logical calculi : Basic Theory of Consequence Operations. Vol. 199. Synthese Library. Kluwer Academic Publishers. ISBN: 978-90-277-2785-5.

Wojtylak, P. (2004). "On a problem of H. Friedman and its solution by T. Prucnal". In: Reports on Mathematical Logic 38, pp. 69-86.

Wroński, A. (1973). "Intermediate Logics and the Disjunction Property". In: Reports on Mathematical Logic 1, pp. 39-51. 